\title{
Adquisición de tecnología biomédica en IPS colombianas: comparación y mejores prácticas*
}

\section{Acquisition of Biomedical Technology in Colombian IPS: Comparison and Best Practices}

\section{Aquisição de tecnologia biomédica nas IPS colombianas: comparação e melhores práticas}

Fecha de recepción: 24-02-16 Fecha de aceptación: 27-08-16 Disponible en línea: 30-11-16 doi:10.11144/Javeriana.rgyps15-31.atbi

Cómo citar este artículo:

Salazar-Flórez KJ, Botero-Botero S, Jiménez-Hernández CN. Adquisición de tecnología biomédica en IPS colombianas: comparación y mejores prácticas Rev. Gerenc. Polít. Salud. 2016; 15(31): 88-118. http:// dx.doi.org/10.11144/Javeriana.rgyps15-31.atbi

Kelly Johanna Salazar-Flórez**

Sergio Botero-Botero***

Claudia Nelcy Jiménez-Hernández $* * * *$

Artículo de investigación resultado de la tesis de Maestría en Ingeniería-Ingeniería Administrativa de la Universidad Nacional de Colombia, Contribución metodológica para la evaluación de la tecnología biomédica en la Clínica Universitaria Bolivariana con fines de acreditación según los estándares de la Joint Commission International. Año de finalización: 2015. Fue realizado por la autora principal bajo la dirección de los coautores.

** $\quad$ M.Sc. en Ingeniería Administrativa, Facultad de Minas, Universidad Nacional de Colombia, Medellín, Colombia Bioingeniera, Jefe de Ambiente Físico y Medio Ambiente en la ESE Hospital Manuel Uribe Ángel de Envigado. Dirección: Diagonal 31 No. 36A Sur 80, Loma de Las Brujas, Envigado, Antioquia. Teléfono fijo: 33948 00, extensión 148. Correo electrónico: kjsalazarf@unal.edu.co

**** Ph.D. en Ciencias, M.Sc. en Ciencias de Ingeniería. Profesor de la Facultad de Minas, Universidad Nacional de Colombia, Medellín, Colombia. Correo electrónico: sbotero@unal.edu.co

**** Ph.D. en Ingeniería, M.Sc. en Administración, profesora de la Facultad de Ciencias Agrarias, Universidad Nacional de Colombia, Bogotá, Colombia. Correo electrónico: cnjimenezh@unal.edu.co 


\section{Resumen}

En este artículo de investigación se analizó el proceso de adquisición tecnológica en las instituciones prestadoras de servicios de salud (IPS) en Colombia. Para este propósito se aplicó una metodología de diagnóstico empresarial denominada benchmarking en seis IPS del departamento de Antioquia (tanto del sector público como del sector privado), lo que permitió identificar las principales formas de adquirir tecnología biomédica, la priorización de las necesidades tecnológicas, los métodos empleados por las instituciones hospitalarias para la evaluación del equipamiento médico, las fuentes de identificación de necesidades, los requisitos legales y técnicos exigidos en el momento de la compra, así como la proporción de búsqueda de alertas nacionales e internacionales en bases de datos relacionadas con los dispositivos. De esta manera, se desarrolló un ejercicio de referenciación comparativa y competitiva como herramienta de gestión que promueve la caracterización e incorporación de las posibles mejores prácticas ejercidas por las clínicas y hospitales del país.

Palabras clave: adquisición tecnológica; benchmarking; equipamiento biomédico; evaluación de tecnologías en salud; organizaciones en salud; tecnología biomédica

\section{Abstract}

We analyzed in this research article the process of technology acquisition in Colombian health service provider institutions (IPS). For this purpose we applied a business assessment methodology called benchmarking in six different IPS of the Antioquia department (both in the public and private sectors). This allowed us to identify the main ways to acquire biomedical technology; the prioritization of technological needs; the methods used by hospitals to assess medical equipment; the sources for the identification of needs; the legal and technical requirements requested at the moment of purchase; and also the amount of searches performed regarding national and international alerts in databases related to the devices. In this way, we developed a comparative and competitive referencing work as a management tool promoting characterization and the inclusion of the possible practical improvements carried out by clinics and hospitals across the country.

Keywords: technology acquisition; benchmarking; biomedical equipment; health technology assessment; health organizations; biomedical technology

\section{Resumo}

Neste artigo de pesquisa foi analisado o processo de aquisição de tecnologia nas instituições que prestam serviços de saúde (IPS, pelas suas siglas em espanhol) na Colômbia. Para o efeito, foi aplicada uma metodologia de diagnóstico empresarial chamada de benchmarking em seis IPS do departamento de Antioquia (tanto do sector público como do privado), o que permitiu identificar as principais maneiras de adquirir tecnologia biomédica, a priorização das necessidades de tecnologia, métodos empregados pelos hospitais para avaliação de equipamentos médicos, fontes de identificação das necessidades, requerimentos legais e técnicos necessários no momento da compra, assim como a proporção de procura de alarmes nacionais e internacionais em bases de dados relacionadas com dispositivos. Assim, um exercício de avaliação comparativa e competitiva foi desenvolvido como ferramenta de gestão que promove a caracterização e incorporação da possível melhor prática exercida por clínicas e hospitais do pais.

Palavras-chave: aquisição de tecnologia; benchmarking; análise comparativa; equipamento biomédico; avaliação de tecnologias de saúde; organizações de saúde; tecnologia biomédica 


\section{Introducción}

La adquisición de tecnología biomédica es el proceso mediante el cual se obtienen los equipos y los dispositivos que se requieren en la institución de salud, según lo que se ha establecido previamente en la planeación. Esta puede efectuarse a través de la compra directa de bienes o servicios, donaciones, alquiler o renta de equipos, contratación, entre otras, e incluye todas las acciones encaminadas a la planificación, identificación de las necesidades y los proveedores, la solicitud, la evaluación, la revisión y la adjudicación de las ofertas, la contratación y todas sus fases de administración del contrato hasta la entrega del bien o servicio. Si el proceso de adquisición o contratación comprende la instalación y puesta en funcionamiento de la tecnología biomédica, puede denominarse "incorporación de tecnología" (1).

La principal función de la adquisición de tecnología biomédica es la de proveer a la institución de salud de equipamiento apropiado, costo-efectivo y seguro para los usuarios y operadores, y que a su vez cumpla con los requisitos de calidad definidos por la organización y la práctica clínica (2).

La toma de decisiones durante el proceso de adquisición de tecnología biomédica se ve afectada por diversos factores como:

- Las nuevas tecnologías médicas, de incuestionable eficacia diagnóstica o terapéutica, se están incorporando de forma progresiva a la asistencia sanitaria. Los decisores que realizan los procesos de adquisición únicamente tienen en cuenta como variable predominante el costo y cómo controlarlo (3).

- Las nuevas tecnologías emergen rápida90 mente y están siendo incorporadas en las organizaciones mucho antes de que pue- da evaluarse rigurosamente su impacto clínico real, sus consecuencias éticas y su impacto económico y social (4).

- La información con la que se evalúan las opciones tecnológicas es imprecisa e insuficiente, lo que dificulta el proceso de decisión (5). Esto se refleja en inversiones de alta o mediana cuantía que al poco tiempo no responden a las necesidades clínicas y epidemiológicas de la comunidad que se atiende, y en el peor de los casos son incompatibles con la infraestructura hospitalaria, lo cual genera altos gastos de mantenimiento e incidentes por mal uso, debido a la falta de entrenamiento y al poco impacto funcional entre los usuarios (6, p. 2).

El último factor considera un conjunto de variables que son críticas en el proceso de evaluación de la tecnología biomédica para su incorporación, y es que si la organización no cuenta con una metodología estandarizada, se va a adquirir el equipamiento con ciertas funcionalidades y características que, debido a los procesos de innovación y al entorno competitivo en el que la organización se desenvuelve, pronto dejará de ser útil para el uso que fue diseñado. Estos cambios en algunas ocasiones implican reemplazar la tecnología adquirida recientemente por nuevos productos del mercado, sin establecer una adecuada relación costo-beneficioseguridad-efectividad.

Con el propósito de estandarizar sus procesos, las IPS han adoptado algunas prácticas clínicas e incluso han llegado a implementar sistemas de gestión de la calidad encaminados a proporcionar los lineamientos y directrices organizacionales relacionados con la seguridad del paciente y las instalaciones físicas, la oportunidad en la atención, el cuidado de la salud de los usuarios y la humanización de los servicios asistenciales. 
El objetivo de este artículo es analizar la adquisición de tecnología biomédica y caracterizar las metodologías de evaluación empleadas en el interior de algunas IPS colombianas pertenecientes a la región antioqueña, para lo cual se realizó un ejercicio de referenciación comparativa en seis instituciones hospitalarias, buscando aportar información que amplíe la comprensión de este proceso en el país, teniendo en cuenta que existe poca evidencia científica para apoyar la toma de decisiones alrededor de la evaluación de los dispositivos, y que es importante el fortalecimiento de la gestión de las necesidades tecnológicas en las organizaciones.

A través de este ejercicio de referenciación comparativa se busca comprobar cómo las instituciones del sector hospitalario han hecho avances significativos en el proceso de adquisición de la tecnología biomédica, mediante el mejoramiento continuo, el incremento de sus capacidades para producir resultados orientados al logro y al aprendizaje permanente sobre lo que realmente saben hacer bien, contribuyendo así a la potenciación de sus experiencias, la transformación de la sociedad, la identificación de los factores críticos de éxito, y a promover la adopción de estos aprendizajes en nuevos entornos organizacionales.

El artículo se encuentra estructurado en cinco secciones, la primera asociada a la conceptualización y a aspectos relevantes de los procesos de adquisición tecnológica; la segunda sección hace referencia al método y los materiales empleados en la investigación, en cuanto a la ejecución de la referenciación comparativa y la aplicación del análisis de resultados; en la tercera sección se presentan los resultados, enfatizando en las diversas metodologías implementadas en la evaluación del equipamiento médico en las IPS. En la siguiente sección se realiza la discusión de los resultados obtenidos, mediante la comparación del proceso de adquisición de la tecnología biomédica efectuado tanto en IPS públicas como en privadas en estudios similares. En la sección final se profundiza en las conclusiones generales del estudio, así como en las mejores prácticas que fueron identificadas a través de la referenciación comparativa.

\section{Aspectos relevantes en la adquisición de tecnología biomédica}

La adquisición de la tecnología biomédica en el ambiente hospitalario:

\begin{abstract}
[...] tiene como propósito el mejoramiento de la eficiencia y la calidad en la prestación de los servicios de salud, por medio de la destinación de limitados recursos a inversiones en tecnología, que brinden mayores beneficios económicos y alta efectividad en los servicios. (6)
\end{abstract}

Para conocer la importancia del proceso de adquisición del equipamiento médico, es necesario situarse en el ciclo de vida de la tecnología sanitaria, que según la Organización Mundial de la Salud (OMS) está comprendido por tres fases. En la figura $1 \mathrm{se}$ observan las tres fases del ciclo de vida de los dispositivos médicos y su interrelación. Estas pueden definirse como $(1,7)$ :

1. Suministro: en esta fase debe aplicarse la política nacional sobre investigación y desarrollo de tecnologías médicas y las necesidades de la población identificadas en relación con la salud, lo cual favorece los trabajos de I+D (innovación + desarrollo) en ciencia e ingeniería.

2. Adquisición: consiste en la evaluación de las tecnologías y los dispositivos, la 
planificación y el análisis de necesidades, así como la adquisición, la instalación y la puesta en funcionamiento.

3. Utilización: es el seguimiento al funcionamiento de la tecnología que se realiza posteriormente a su adquisición, así como el entrenamiento, el mantenimiento y el retiro del servicio.

Antes de realizar la adquisición de la tecnología biomédica, es fundamental efectuar una evaluación previa de las necesidades institucionales y del equipamiento que se va a incorporar. Esta actividad consiste en la determinación y la definición de los dispositivos médicos y su clasificación en función del grado de prioridad. Para esto se deben considerar los objetivos generales o lineamientos estratégicos de la institución, las instalaciones y la infraestructura existente, los planes a corto, mediano y largo plazo y el nivel de desarrollo del recurso humano, antes de proceder a la compra del dispositivo médico. Esta evaluación debe efectuarse en el marco de un programa eficaz de mantenimiento preventivo de equipos médicos, cuando se actualicen los inventarios de los activos fijos, se reevalúen los servicios o se sustituya la tecnología biomédica. Las necesidades tecnológicas también deben ser analizadas antes de la construcción de una nueva institución de salud (8).

En algunas instituciones se emplean "evaluaciones de necesidades de equipos biomédicos" con el fin de promover la recolección de la información por cada servicio asistencial. Estas herramientas contribuyen a la identificación del conjunto de equipos que son necesarios para ser adquiridos por un área específica o centro de costo.

Además de lo anterior, es fundamental que cada institución de salud defina su propia política de adquisición de tecnología biomédica, estableciendo las directrices del plan de compras de los dispositivos, según las necesidades, las prioridades y la disponibilidad de los recursos.

Sin embargo, la planificación de la sustitución de los equipos es la que garantiza la seguridad de los usuarios y operarios de la tecnología, proporcionando resultados confiables y minimizando el costo de las reparaciones de los dispositivos obsoletos, los cuales en la mayoría de casos ya no se encuentran cubiertos por el servicio técnico (1).

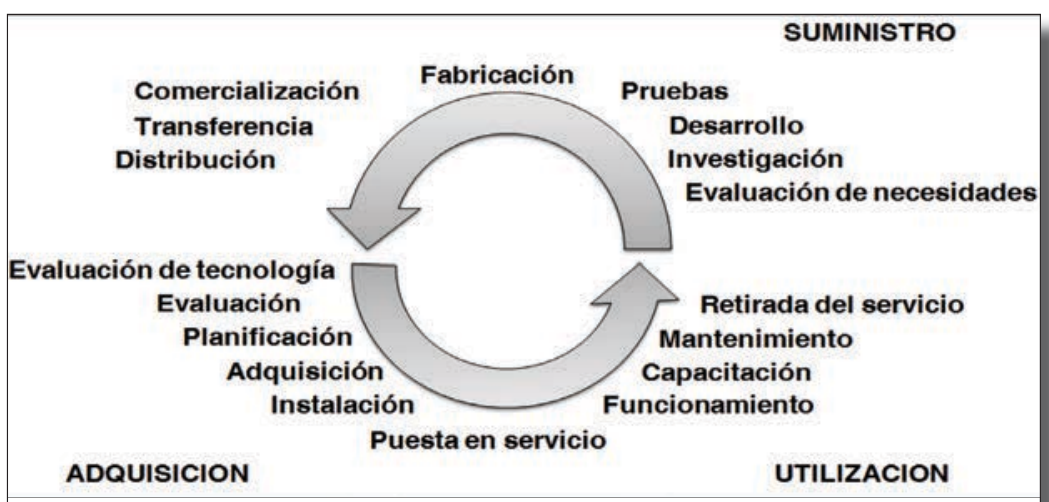

Figura 1. Fases del CiClo de VIDa de la tecnología SANitaria

Fuente: Organización Mundial de la Salud (7) 
La planeación estratégica de las necesidades tecnológicas debe incluir la renovación del equipamiento médico, la reposición y el proceso de baja.

- Renovación de tecnología biomédica: se realiza teniendo en cuenta los planes y programas de mantenimiento, evaluando el estado físico-funcional de la tecnología con respecto a su seguridad, eficiencia y productividad; la disponibilidad de los recursos financieros, evitando que los equipos se vuelvan obsoletos y por consiguiente se presenten problemas de accesibilidad y oportunidad en la prestación de los servicios de salud (7).

La renovación se puede efectuar con dos propósitos: mejorar el estado del equipamiento que se tiene en la institución, o introducir nueva tecnología que aún no ha sido implementada, para ampliar la cobertura del portafolio de servicios.

- Reposición de tecnología biomédica: es la actividad que debe responder a situaciones de emergencia, optimizando el recurso financiero. Esta puede presentarse por varias causas:

- Los equipos biomédicos están dañados y no pueden repararse.

- Se encuentran depreciados, lo que deriva en altos costos de mantenimiento, ocasionados por las numerosas intervenciones de la tecnología.

- El equipamiento biomédico ha sido dado de baja.

- La tecnología biomédica representa un riesgo para los operadores y/o usuarios durante su utilización o es insuficiente para atender la frecuente demanda del servicio asistencial (9).
Estas condiciones originan una pérdida de eficiencia del equipo, lo cual incrementa los costos significativamente. A su vez, las políticas se encuentran directamente relacionadas con el proceso de baja de los dispositivos médicos.

- Baja de la tecnología: es el proceso mediante el cual el equipo biomédico es retirado del servicio porque se encuentra obsoleto, es decir, está en desuso, no por su mal funcionamiento, sino por un insuficiente desempeño de sus funciones en comparación con los nuevos equipos y tecnologías introducidos en el mercado.

Es entonces cuando se determina que el equipo ha cumplido su tiempo de vida útil -duración estimada en tiempo en la que puede desempeñar correctamente las funciones para las cuales ha sido diseñadoespecificada por el fabricante. La tecnología biomédica también se da de baja cuando su pérdida o siniestro produce su salida del inventario como activo fijo (6).

Una vez que se tienen identificadas todas las necesidades de tecnología biomédica, es necesario jerarquizarlas para determinar su priorización o el orden en que deben atenderse.

El proceso de priorización de las necesidades, la generación de políticas y la implementación de iniciativas de adquisición tecnológica en salud deben ser formulados según el perfil epidemiológico de la región, el país o el continente. Los cambios en el patrón de enfermedades y diferencias epidemiológicas entre regiones y países deben ser incorporados para la elaboración de las listas del equipamiento biomédico requerido, las cuales se derivan a su vez de los paquetes esenciales de salud (10). 
No siempre se cuenta con los recursos financieros necesarios para atender todas las necesidades tecnológicas. Por lo tanto, deben considerarse múltiples opciones, asignando una valoración a cada una de estas para identificar aquellas que son prioritarias y que a su vez proporcionen respuesta a ciertos aspectos clave, tales como: el impacto ejercido sobre la población, el incremento de la cobertura en la prestación de los servicios de salud, así como la aceptabilidad y la disponibilidad de los recursos institucionales.

\section{Materiales y métodos}

El estudio realizado es de tipo descriptivo y exploratorio, desarrollado con información procedente de fuentes primarias que se obtuvo a través del diseño y la aplicación de un instrumento de consulta, como se detalla más adelante, y de fuentes secundarias como informes y documentos relacionados con las IPS estudiadas. Se emplearon técnicas cualitativas y cuantitativas basadas en la referenciación comparativa o benchmarking. En la figura 2 se presenta la metodología empleada para realizar el diagnóstico de los hospitales y clínicas del departamento de Antioquia, con el fin de establecer los factores críticos de éxito e identificar las posibles mejores prácticas en el proceso de adquisición de tecnología biomédica. A continuación se describen las actividades ejecutadas en cada una de las fases:

\section{Fase 1. Delimitación de la población y muestra}

La población definida para efectuar la investigación la constituye el conjunto de IPS, tanto públicas como privadas, del departamento de Antioquia, que han sido reconocidas durante los años 2013 y 2014 por su trayectoria y participación en los diferentes proyectos, premios y/o galardones que ha celebrado el sector hospitalario. Estas IPS se encuentran acreditadas según los estándares nacionales e internacionales de calidad en salud y ocuparon las mejores posiciones en el ranking de hospitales y clínicas de Latinoamérica (11).

Para la selección de las tres instituciones públicas y las tres pertenecientes al sector privado, se realizó una matriz de correlación, donde se asignaron puntajes para determinar el impacto total por reconocimiento (ITPR) (12). Este se estimó al valorar cada certificación (reconocimiento) obtenida, mediante 0 o 1 , teniendo en cuenta que estas impactan sobre el good will de las organizaciones. Posteriormente, estas calificaciones se sumaron entre sí, buscando una correlación entre las certificaciones, los

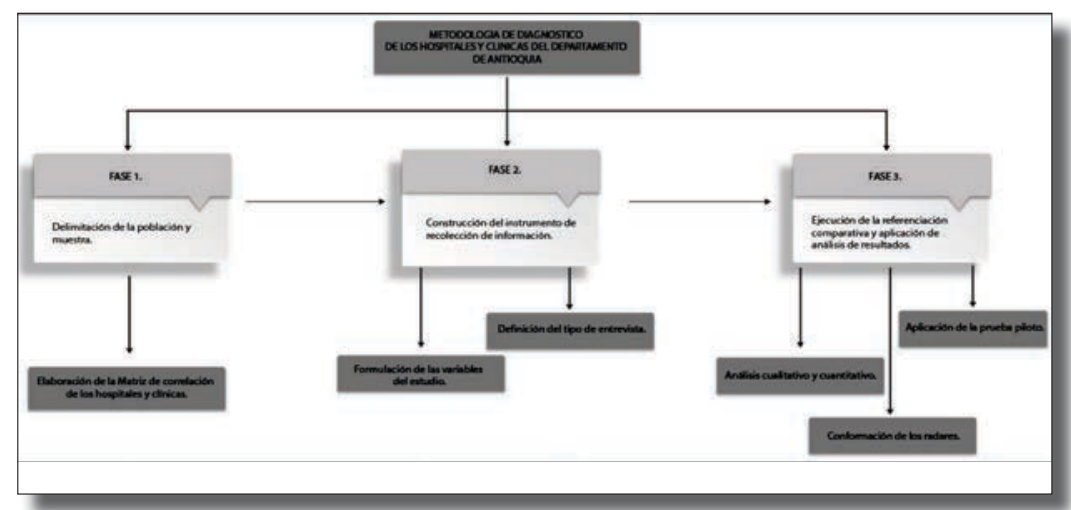


objetivos estratégicos y los factores críticos para cada IPS del estudio. Se definió el universo geográfico del departamento antioqueño, porque es una región caracterizada por el creciente desarrollo del sector hospitalario y por la posibilidad de acceder a las entrevistas y conocer de primera mano el proceso de adquisición de tecnología biomédica.

En la tabla 1 se presentan y se describen de manera general los proyectos, los premios y los galardones del sector hospitalario, y que fueron tomados como referencia para la selección de las clínicas y los hospitales de la muestra (13).

\section{Fase 2. Construcción del instrumento de recolección de información (encuesta)}

Se elaboró un conjunto formalizado de preguntas, para lo cual se tuvieron en cuenta las pautas de formulación de cuestionarios y formatos para investigación de mercados (14). Esto hizo parte de la estrategia de recolección de datos, ya que también se emplearon otros métodos para obtener información, como la revisión documental de procedimientos y registros de las organizaciones. Para minimizar el error de respuesta se introdujeron preguntas estructuradas y preguntas no estructuradas. Las preguntas

Tabla 1. Criterios de SElección de las instituciones PRESTAdoras de SERVICIOS DE SALud

\begin{tabular}{|c|c|c|}
\hline Proyecto/premio/galardón & Descripción & $\begin{array}{l}\text { Responsable del } \\
\text { otorgamiento }\end{array}$ \\
\hline $\begin{array}{l}\text { Proyecto de referenciación } \\
\text { comparativa para indicadores } \\
\text { en gestión de tecnología } \\
\text { biomédica y técnicas de } \\
\text { producción limpia (2012) }\end{array}$ & $\begin{array}{l}\text { Este proyecto se realizó para identificar las } \\
\text { mejores prácticas de las instituciones hospitalarias } \\
\text { colombianas en relación con la gestión de la } \\
\text { tecnología biomédica, el consumo de servicios } \\
\text { públicos y la gestión integral de residuos } \\
\text { hospitalarios. }\end{array}$ & $\begin{array}{l}\text { Asociación } \\
\text { Colombiana de } \\
\text { Hospitales y } \\
\text { Clínicas }\end{array}$ \\
\hline $\begin{array}{l}\text { Premio a la Calidad Medellín } \\
\text { ciudad saludable (2013-2014) }\end{array}$ & $\begin{array}{l}\text { Este premio busca generar conciencia sobre la } \\
\text { responsabilidad que tienen los diferentes actores } \\
\text { de la ciudad de Medellín en el mejoramiento de las } \\
\text { condiciones de salud y bienestar de la población, } \\
\text { en la contribución al desarrollo humano integral y } \\
\text { la calidad de vida, en la responsabilidad de prestar } \\
\text { servicios de salud humanizados, seguros, donde se } \\
\text { fomente la promoción, la educación, la prevención } \\
\text { de la salud y el control de factores de riesgo. }\end{array}$ & $\begin{array}{l}\text { Alcaldía de } \\
\text { Medellín }\end{array}$ \\
\hline $\begin{array}{l}\text { Galardón Hospital Seguro } \\
(2013-2014)\end{array}$ & $\begin{array}{l}\text { Este galardón propende por el reconocimiento } \\
\text { del trabajo permanente de las clínicas y los } \\
\text { hospitales del país, para brindar servicios de salud } \\
\text { a los colombianos, soportados en altos estándares } \\
\text { de seguridad para los pacientes. }\end{array}$ & $\begin{array}{l}\text { Alcaldía de } \\
\text { Medellín }\end{array}$ \\
\hline $\begin{array}{l}\text { Acreditación según } \\
\text { estándares nacionales de } \\
\text { calidad en salud (2014) }\end{array}$ & $\begin{array}{l}\text { La adopción del modelo de acreditación en salud } \\
\text { colombiano es de carácter voluntario, pero ha } \\
\text { cobrado mucha importancia porque constituye la } \\
\text { base para que las instituciones hospitalarias evalúen } \\
\text { la necesidad de cumplir requisitos que trasciendan las } \\
\text { obligaciones legales básicas. }\end{array}$ & Icontec \\
\hline
\end{tabular}




\begin{tabular}{|c|c|c|}
\hline Proyecto/premio/galardón & Descripción & $\begin{array}{l}\text { Responsable del } \\
\text { otorgamiento }\end{array}$ \\
\hline $\begin{array}{l}\text { Acreditación con estándares } \\
\text { internacionales de calidad } \\
\text { en salud, según la Joint } \\
\text { Commission International } \\
(2014)\end{array}$ & $\begin{array}{l}\text { La implementación de estos estándares } \\
\text { internacionales para la acreditación es de carácter } \\
\text { voluntario y permite a las instituciones enfrentarse } \\
\text { al establecimiento de protocolos y acciones } \\
\text { encaminados hacia el mejoramiento continuo y la } \\
\text { excelencia en la atención. }\end{array}$ & $\begin{array}{l}\text { Joint } \\
\text { Commission } \\
\text { International }\end{array}$ \\
\hline $\begin{array}{l}\text { Ranking de hospitales y } \\
\text { clínicas de Latinoamérica } \\
(2014)\end{array}$ & $\begin{array}{l}\text { Busca el reconocimiento de las instituciones } \\
\text { hospitalarias de América Latina, en relación } \\
\text { con el desempeño y los resultados obtenidos en } \\
\text { sus indicadores de procesos. Se evalúa también } \\
\text { la capacidad instalada, el capital humano, } \\
\text { el prestigio de la institución y la gestión del } \\
\text { conocimiento. }\end{array}$ & $\begin{array}{l}\text { Revista América } \\
\text { Economía }\end{array}$ \\
\hline $\begin{array}{l}\text { Premio a la Gestión } \\
\text { Transparente Antioquia Sana } \\
(2013-2014)\end{array}$ & $\begin{array}{l}\text { Este premio tiene como objetivo principal } \\
\text { reconocer el ejercicio ético y legal de los procesos } \\
\text { realizados por las empresas sociales del Estado } \\
\text { en el manejo transparente de los recursos de } \\
\text { la salud, con el propósito de fortalecer sus } \\
\text { capacidades para contribuir a la competitividad } \\
\text { y el crecimiento sostenible de la organización, lo } \\
\text { que redundará en el desarrollo del departamento } \\
\text { de Antioquia. }\end{array}$ & $\begin{array}{l}\text { Gobernación de } \\
\text { Antioquia }\end{array}$ \\
\hline
\end{tabular}

Fuente: elaboración propia

estructuradas "especifican el conjunto de alternativas de respuesta y su formato. Una pregunta estructurada puede ser de opción múltiple, dicotómica o una escala". Estas se clasifican como se indica en la tabla 2.

Las preguntas no estructuradas o abiertas son muy útiles como preguntas iniciales sobre un tema en una investigación exploratoria, porque tienden a sesgar menos la respuesta que las preguntas estructuradas (16). En la construcción de la encuesta aplicada se emplearon preguntas estructuradas (de opciones múltiples y dicotómicas), preguntas de frecuencia, preguntas marca-puntaje y preguntas no estructuradas.
- Formulación de las variables de la encuesta: se definieron nueve variables relacionadas con los cuatro temas o conceptos principales asociados a la adquisición de tecnología biomédica, según se observa en la tabla 3; así mismo, se presenta su correspondencia con cada pregunta que se formuló en el cuestionario.

- Definición del tipo de entrevista: existen tres tipos de métodos de entrevista que influyen en el diseño del cuestionario: las entrevistas personales, las telefónicas y las entrevistas por correo electrónico. Para lograr recolectar la mayor información posible, se aplicó la entrevista de tipo personal a los diferentes expertos 
Tabla 2. Clasificación de las PReguntas Según SU Tipología

\begin{tabular}{|l|l|}
\hline $\begin{array}{l}\text { Tipo de } \\
\text { preguntas }\end{array}$ & Descripción \\
\hline Estructuradas & $\begin{array}{l}\text { Preguntas de opción múltiple: en este tipo de preguntas el investigador ofrece las } \\
\text { opciones de las respuestas y se solicita al encuestado que seleccione una o más de } \\
\text { las alternativas propuestas (14). } \\
\text { Preguntas dicotómicas: en este tipo de preguntas el encuestado solo tiene dos } \\
\text { alternativas de respuesta: sí o no, de acuerdo o en desacuerdo, entre otras. En algunas } \\
\text { ocasiones las dos alternativas de interés se complementan con una alternativa } \\
\text { neutral, como por ejemplo sin opinión, sin conocimiento, no lo sé, o ninguna (14). } \\
\text { Preguntas de escalas: la medición por escalamiento es la asignación de números, } \\
\text { valores o símbolos a características del tema de investigación, de acuerdo con un } \\
\text { conjunto de reglas previamente definidas. La técnica de escalamiento se clasifica } \\
\text { como comparativa y no comparativa (14). }\end{array}$ \\
\hline Frecuencia & $\begin{array}{l}\text { Estas preguntas permiten determinar la periodicidad con la cual la empresa lleva a } \\
\text { cabo determinados procesos o actividades (15). }\end{array}$ \\
\hline Marca-puntaje & $\begin{array}{l}\text { Son preguntas donde la mejor situación está determinada por el mayor número de } \\
\text { respuestas seleccionadas (por ejemplo, si de seis opciones existentes en la pregunta } \\
\text { se marcan las seis, se obtiene un cinco como puntaje) (15). }\end{array}$ \\
\hline
\end{tabular}

Fuente: elaboración propia con base en (14) y (15)

Tabla 3. Formulación de las variables y PReguntas de la enCuesta

\begin{tabular}{|l|l|l|}
\hline Concepto & Variables objeto de medición & $\begin{array}{l}\text { Orden jerárquico y } \\
\text { equivalencia de las preguntas } \\
\text { según el tipo de variables }\end{array}$ \\
\hline $\begin{array}{l}\text { Adquisición de } \\
\text { nuevas tecnologías } \\
\text { biomédicas }\end{array}$ & $\begin{array}{l}\text { Variable } V_{1} \text {, formas de adquirir tecnología: facilita la } \\
\text { identificación de las diversas formas por medio de las cuales } \\
\text { se puede adquirir una tecnología. Si no se cuenta con una } \\
\text { planeación estratégica del equipamiento, es difícil que el } \\
\text { proceso de explotación de esta sea sostenible. El proceso de } \\
\text { adquisición no se debe llevar a cabo hasta que no se haya } \\
\text { realizado una planificación tecnológica adecuada y acorde } \\
\text { con las necesidades organizacionales }(17) .\end{array}$ & $\begin{array}{l}\text { La variable } V_{1} \text { corresponde a } \\
\text { las preguntas } 1 \text { y } 2 .\end{array}$ \\
$\begin{array}{l}\text { Variable } V_{2,} \text { planificación de la adquisición de tecnología: para } \\
\text { proveer a las IPS de la tecnología apropiada, costo-efectiva } \\
\text { y segura para los usuarios y los colaboradores, y que además } \\
\text { cumpla con los requisitos técnicos de calidad establecidos } \\
\text { por la organización y por la práctica internacional, se debe } \\
\text { llevar a cabo una adecuada planeación de esta (9). }\end{array}$ & $\begin{array}{l}\text { La variable } V_{2} \text { hace referencia } \\
\text { a las preguntas 3, } 4 \text { y 5. }\end{array}$ \\
\hline $\begin{array}{l}\text { Evaluación de } \\
\text { las necesidades } \\
\text { de adquisición } \\
\text { de tecnología } \\
\text { biomédica }\end{array}$ & $\begin{array}{l}\text { Variable } V_{3,} \text { priorización de necesidades de adquisición de } \\
\text { tecnología biomédica: la evaluación de necesidades de } \\
\text { adquisición debe estar centrada en políticas de reposición } \\
\text { y renovación de la tecnología biomédica y en un sistema } \\
\text { de prioridades vinculado con la función misional de la } \\
\text { institución de salud. Estas políticas deben ser formuladas } \\
\text { de manera conjunta con la dirección estratégica de la } \\
\text { organización (18). }\end{array}$ & $\begin{array}{l}\text { La variable } V_{3} \text { está } \\
\text { correlacionada con las } \\
\text { preguntas 6, } 7,8,9 \mathrm{y} 10 .\end{array}$ \\
\hline
\end{tabular}




\begin{tabular}{|c|c|c|}
\hline Concepto & Variables objeto de medición & $\begin{array}{l}\text { Orden jerárquico y } \\
\text { equivalencia de las preguntas } \\
\text { según el tipo de variables }\end{array}$ \\
\hline $\begin{array}{l}\text { Gestión del } \\
\text { proceso de } \\
\text { adquisición }\end{array}$ & 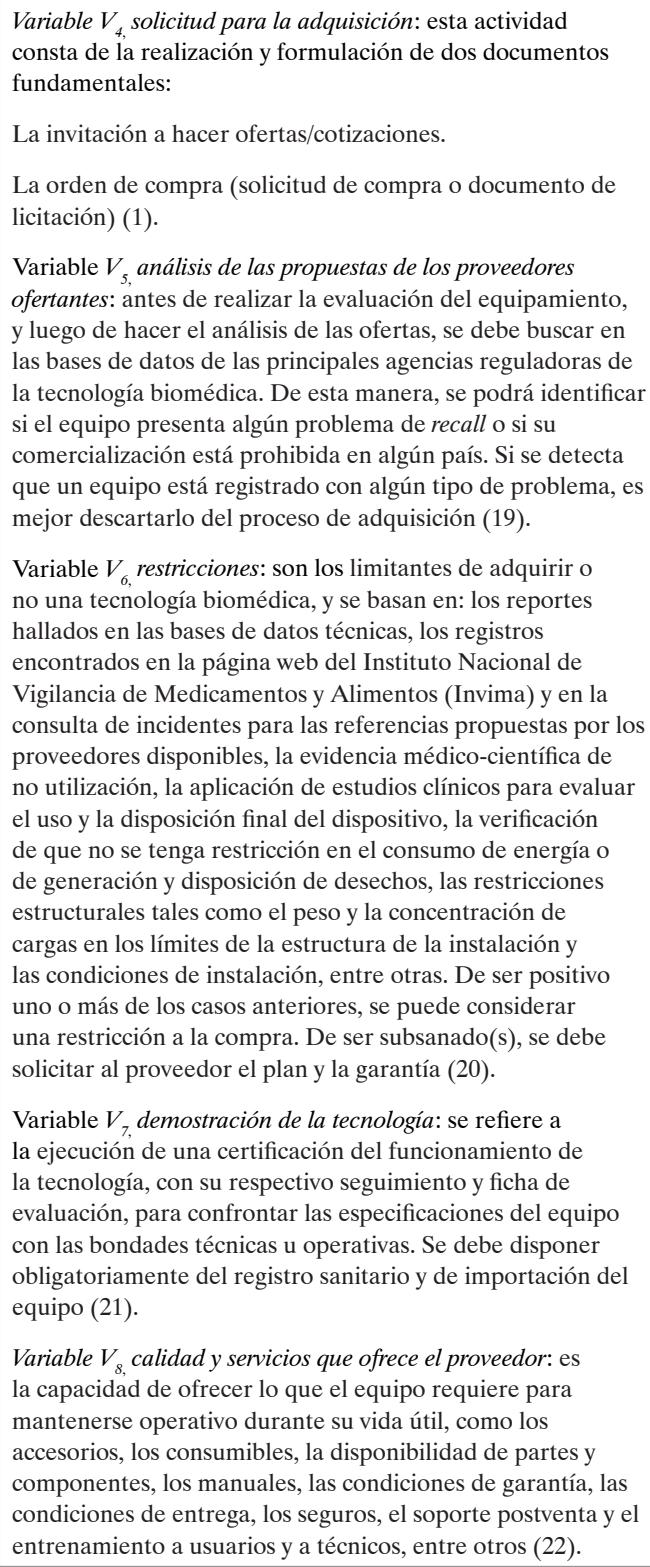 & $\begin{array}{l}\text { La variable } V_{4} \text { corresponde a } \\
\text { la pregunta } 11 . \\
\text { La variable } V_{5} \text { está } \\
\text { correlacionada con la } \\
\text { pregunta } 12 . \\
\text { La variable } V_{6} \text { hace referencia } \\
\text { a las preguntas } 13 \text { y } 14 \text {. } \\
\text { La variable } V_{7} \text { corresponde a } \\
\text { la pregunta } 15 \text {. } \\
\text { La variable } V_{8} \text { hace referencia } \\
\text { a la pregunta } 16 \text {. }\end{array}$ \\
\hline $\begin{array}{l}\text { Metodologías } \\
\text { para la evaluación } \\
\text { de la tecnología } \\
\text { biomédica que se } \\
\text { desea adquirir }\end{array}$ & $\begin{array}{l}\text { Variable } V_{9} \text { realización de la evaluación y selección final } \\
\text { de la tecnología que será adquirida: permite identificar los } \\
\text { principales métodos en la evaluación de tecnologías, los } \\
\text { cuales incluyen: seguridad, eficacia, efectividad, impacto } \\
\text { organizativo, ético y social, y evaluación económica, entre } \\
\text { otros (23). }\end{array}$ & $\begin{array}{l}\text { La variable } V_{9} \text { corresponde a } \\
\text { las preguntas } 17,18,19 \text { y } 20 .\end{array}$ \\
\hline
\end{tabular}


y líderes relacionados con el proceso de adquisición de tecnología biomédica en las instituciones, porque los encuestados tienen la posibilidad de observar el cuestionario e interactuar directamente con el entrevistador, y esto les permite realizar preguntas largas, complejas y diversas (14).

\section{Fase 3. Ejecución de la referenciación} comparativa y aplicación del análisis de resultados

Durante la referenciación comparativa se entrevistó a los jefes de compras, a los jefes de logística, a los ingenieros biomédicos, a los jefes de ingeniería y mantenimiento, a los jefes de recursos físicos y a los jefes de equipos biomédicos de las instituciones seleccionadas. Para el adecuado análisis de la información obtenida por medio de las encuestas, se efectuó una tabulación de acuerdo con el tipo de pregunta. Es importante aclarar que el valor tomado por cada variable analizada es el resultado de la ponderación de una serie de preguntas tendientes a evaluar el desempeño del proceso de adquisición tecnológica en las organizaciones. Cada una de estas preguntas, dependiendo de su tipo, está sujeta a una cuantificación diferente y determinada por los siguientes parámetros:

- Preguntas dicotómicas cerradas: para las preguntas cuya opción es sí, no, o sin conocimiento, se califica 5 para afirmativo y 0 para negativo o sin conocimiento, tomando 5 como el mayor puntaje obtenido.

- Preguntas abiertas: la finalidad de estas es obtener información con las propias palabras del encuestado y así verificar la consistencia de sus afirmaciones con la conceptualización efectuada, ya que con otro tipo de preguntas las respues- tas podrían estar condicionadas a las presentadas en el formato.

- Preguntas marca-puntaje: la ponderación estará dada por la siguiente fórmula:

$$
P_{\text {preg }}=\left[\frac{F_{c}}{n}\right] * 5
$$

Donde:

$P_{\text {preg }}=$ puntaje obtenido en una pregunta.

$\mathrm{F}_{\mathrm{c}}=$ número de factores considerados por la empresa.

$\mathrm{n}=$ número total de factores que hacen parte de una pregunta.

En la tabla 4 se encuentra la clasificación y el tipo de análisis, de acuerdo con las preguntas del cuestionario.

Una vez ejecutadas las visitas de campo, con su respectiva entrevista y aplicación del instrumento de recolección de información, se empleó el análisis cualitativo, el análisis cuantitativo y la conformación de gráficos de radar para la evaluación de los resultados obtenidos. En la tabla 5 se describe el tipo de análisis y el método de medición implementado.

\section{Resultados}

En esta sección se presentan los resultados obtenidos de la aplicación del instrumento de diagnóstico empresarial en las instituciones hospitalarias que fueron seleccionadas; así mismo, se realiza su respectiva discusión y el análisis. En la primera parte se explica la metodología llevada a cabo para elegir las seis IPS en las cuales se ejecutó la referenciación comparativa, a través de la implementación de una matriz de correlación. Los criterios tenidos en cuenta para su construcción se describen en la tabla 1. 
Kelly Johanna Salazar-Flórez • Sergio Botero- Botero • Claudia Nelcy Jiménez-Hernández

Tabla 4. ClasificaCión de las PREguntas SEgún SU TIPOlogía Y METOdOlogía PaRa ANÁlisis

\begin{tabular}{|c|c|c|}
\hline $\begin{array}{l}\text { Va- } \\
\text { riable }\end{array}$ & Tipo/número de pregunta & $\begin{array}{l}\text { Tipo de } \\
\text { análisis }\end{array}$ \\
\hline$V_{1}$ & $\begin{array}{l}\text { Estructurada } \\
\text { (opción múltiple) / 1a, b, c, d }\end{array}$ & Cuantitativo \\
\hline$V_{1}$ & No estructurada/1e & Cualitativo \\
\hline$V_{1}$ & No estructurada/2 & Cualitativo \\
\hline$V_{2}$ & No estructurada $/ 3$ & Cualitativo \\
\hline$V_{2}$ & $\begin{array}{l}\text { Estructurada } \\
\text { (Opción múltiple)/4a, b, c, d, e }\end{array}$ & Cuantitativo \\
\hline$V_{2}$ & No estructurada/4f & Cualitativo \\
\hline$V_{2}$ & No estructurada $/ 4 \mathrm{~g}$ & Cualitativo \\
\hline$V_{2}$ & $\begin{array}{l}\text { Estructurada } \\
\text { (dicotómica)/5a, b, c }\end{array}$ & Cuantitativo \\
\hline$V_{2}$ & No estructurada/5d & Cualitativo \\
\hline$V_{3}$ & No estructurada/6d & Cualitativo \\
\hline$V_{3}$ & $\begin{array}{l}\text { Estructurada } \\
\text { (Dicotómica)/7a, b, c }\end{array}$ & Cuantitativo \\
\hline$V_{3}$ & No estructurada/7d & Cualitativo \\
\hline$V_{3}$ & $\begin{array}{l}\text { Estructurada (opción } \\
\text { múltiple)/8a, b, c, d, e, f }\end{array}$ & Cuantitativo \\
\hline$V_{3}$ & No estructurada/8g & Cualitativo \\
\hline$V_{3}$ & No estructurada/9 & Cualitativo \\
\hline$V_{3}$ & $\begin{array}{l}\text { Estructurada } \\
\text { (dicotómica)/10a, b, c }\end{array}$ & Cuantitativo \\
\hline$V_{3}$ & No estructurada/10d & Cualitativo \\
\hline$V_{4}$ & $\begin{array}{l}\text { Estructurada } \\
\text { (dicotómica)/11a, b, c }\end{array}$ & Cuantitativo \\
\hline$V_{4}$ & $\begin{array}{l}\text { Estructurada } \\
\text { (Opción múltiple) 11d, e, f, g, } \\
\text { h, i, j, k, l, m, n }\end{array}$ & Cuantitativo \\
\hline
\end{tabular}

\begin{tabular}{|c|l|l|}
\hline $\begin{array}{c}\text { Va- } \\
\text { riable }\end{array}$ & Tipo/número de pregunta & $\begin{array}{l}\text { Tipo de } \\
\text { análisis }\end{array}$ \\
\hline$V_{4}$ & No estructurada/11p & Cualitativo \\
\hline$V_{5}$ & $\begin{array}{l}\text { Estructurada } \\
\text { (dicotómica)/12a, b, c }\end{array}$ & Cuantitativo \\
\hline$V_{5}$ & $\begin{array}{l}\text { Estructurada } \\
\text { (opción múltiple)/12d, e, f, g, h, i,j, } \mathrm{k}\end{array}$ & Cuantitativo \\
\hline$V_{5}$ & No estructurada $/ 12 \mathrm{l}$ & Cualitativo \\
\hline$V_{5}$ & $\begin{array}{l}\text { Estructurada } \\
\text { (dicotómica) } 12 \mathrm{~m}, \mathrm{n}, \mathrm{o}\end{array}$ & Cuantitativo \\
\hline$V_{5}$ & No estructurada/12p & Cualitativo \\
\hline$V_{6}$ & $\begin{array}{l}\text { Estructurada } \\
\text { (Opción múltiple)/13a, b, c, d, e, } \mathrm{f}\end{array}$ & Cuantitativo \\
\hline$V_{6}$ & No estructurada/13g & Cualitativo \\
\hline$V_{6}$ & No estructurada/14 & Cualitativo \\
\hline$V_{7}$ & $\begin{array}{l}\text { Estructurada } \\
\text { (Opción múltiple)/15a, b, c, d, e, f }\end{array}$ & Cuantitativo \\
\hline$V_{7}$ & No estructurada/15g & Cualitativo \\
\hline$V_{8}$ & $\begin{array}{l}\text { Estructurada } \\
\text { (dicotómica)/16a, b, c }\end{array}$ & Cuantitativo \\
\hline$V_{8}$ & No estructurada/16d & Cualitativo \\
\hline$V_{9}$ & No estructurada/17 & Cualitativo \\
\hline$V_{9}$ & No estructurada/18 & Cualitativo \\
\hline$V_{9}$ & No estructurada/19 & Cualitativo \\
\hline$V_{9}$ & $\begin{array}{l}\text { Estructurada } \\
\text { (opción múltiple)/20a, b, c, d, e, } \mathrm{f}\end{array}$ & Cuantitativo \\
\hline$V_{9}$ & No estructurada $/ 20 \mathrm{~g}$ & Cualitativo \\
\hline & & \\
\hline
\end{tabular}

Fuente: elaboración propia

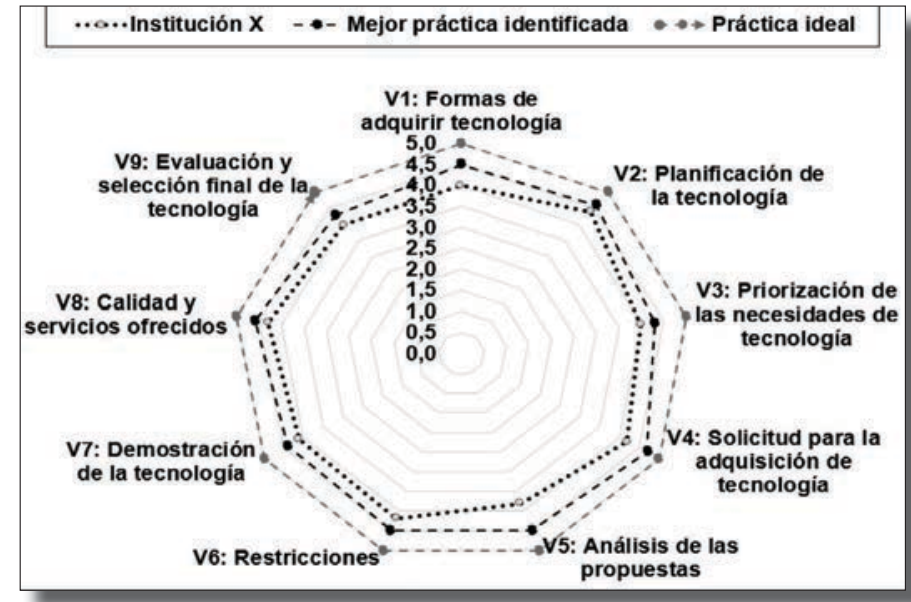




\begin{tabular}{|c|c|}
\hline Tipo de análisis & Método de medición \\
\hline Cualitativo & $\begin{array}{l}\text { Se utilizó el análisis cualitativo para analizar las preguntas no } \\
\text { estructuradas del cuestionario. El enfoque cualitativo no pretende } \\
\text { allegar información para extraer conclusiones representativas } \\
\text { estadísticamente, sino para facilitar el proceso de comprensión e } \\
\text { interpretación de los resultados obtenidos en el análisis cuantitativo. }\end{array}$ \\
\hline Cuantitativo & $\begin{array}{l}\text { Se aplicaron gráficos circulares o de anillos y de tendencias } \\
\text { (histogramas). Las respuestas a cada una de las preguntas del } \\
\text { cuestionario se categorizaron, con el fin de convertirlas en } \\
\text { condiciones cuantificables para efectuar el análisis respectivo. }\end{array}$ \\
\hline $\begin{array}{l}\text { Conformación } \\
\text { de los gráficos de } \\
\text { radar }\end{array}$ & $\begin{array}{l}\text { Para realizar el análisis cuantitativo de las variables del estudio se } \\
\text { efectuaron gráficos de tipo radar con la información resultante de la } \\
\text { ponderación realizada. Los gráficos conformados representan una } \\
\text { institución, y cada una de las variables del estudio constituye un eje } \\
\text { de la figura. } \\
\text { Para establecer la manera como se constituyó cada radar, en la } \\
\text { figura } 3 \text { se presentan los puntajes asignados a las preguntas que } \\
\text { representan las variables del estudio. Estas fueron codificadas a } \\
\text { través de una letra y un número para su respectiva identificación } \\
\text { y el análisis de los resultados. El valor trasladado a cada eje es el } \\
\text { promedio alcanzado en la calificación numérica de las preguntas } \\
\text { que obtuvieron respuesta, asignando una tabla puntaje por cada } \\
\text { institución hospitalaria. }\end{array}$ \\
\hline $\begin{array}{l}\text { Aplicación de la } \\
\text { prueba piloto }\end{array}$ & $\begin{array}{l}\text { Después de determinar el contenido del cuestionario y trabajar } \\
\text { en la estructura de los enunciados, se efectúo una revisión para } \\
\text { verificar que tanto la redacción como la secuencia de las preguntas } \\
\text { se encontraran acordes con los objetivos de la investigación. } \\
\text { Posteriormente, el cuestionario revisado fue sometido a una prueba } \\
\text { piloto por un experto (jefe de ingeniería y mantenimiento) de la } \\
\text { Clínica Universitaria Bolivariana, que consistió en la aplicación de } \\
\text { un pre-test de este, donde se cronometró el tiempo que tardaba en } \\
\text { responderlo, y de esta forma estimar el tiempo promedio para su } \\
\text { ejecución. También se evaluaron la consistencia y la concordancia } \\
\text { de las preguntas formuladas. }\end{array}$ \\
\hline
\end{tabular}

Fuente: elaboración propia

Para la construcción de la matriz de correlación, se asignó un punto - 1 o 0 - por cada reconocimiento obtenido durante la participación en proyectos, premios y/o galardones en el área de la salud, ocupar las mejores posiciones en el ranking de hospitales y clínicas, o haber alcanzado la acreditación nacional o internacional. En la tabla 6 se mencionan en orden alfabético las instituciones y el ITPR, el cual corresponde a la sumatoria de todos los puntos obtenidos en cada uno de los ítems. Los hospitales y las clínicas que obtuvieron mayor puntaje fueron seleccionados para la ejecución de la referenciación comparativa. 
En la tabla 6 se observa que el mayor ITPR fue obtenido por siete instituciones, tres del sector público y cuatro del sector privado. El instrumento de diagnóstico fue aplicado a las siete organizaciones, aclarando que dentro del estudio no se tienen en cuenta los resultados de la Clínica Universitaria Bolivariana, por solicitud de la institución. Una vez seleccionadas las IPS para efectuar las entrevistas, se acordaron las fechas con cada organización para realizar la visita a las instalaciones y aplicar el instrumento de diagnóstico empresarial a los diferentes expertos y líderes. Con el propósito de facilitar la discusión y el análisis de los resultados, se empleó una letra para identificar a las entidades. Por consiguiente, se analizaron los resultados obtenidos para cada una de las variables del estudio con el fin de caracterizar las metodologías empleadas en las IPS. La primera variable es $V_{1}$ (formas de adquirir tecnología). En la figura 4 se observa que $l a$ adquisición según la asignación presupuestal es la principal forma empleada en el momento de comprar tecnología biomédica en las seis IPS de la muestra, con un porcentaje del $67 \%$, seguida de la opción del comodato, para el que se obtuvo un $33 \%$.

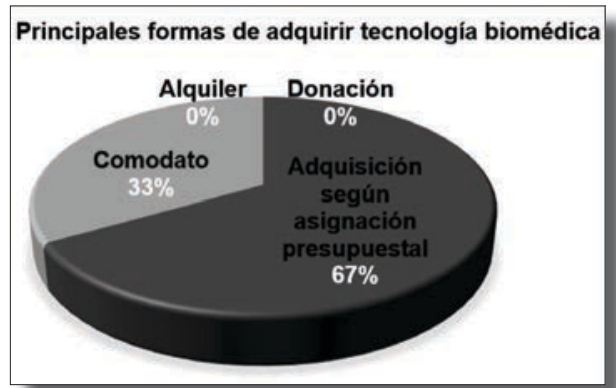

Figura 4. Resultados para la variable $V_{l}$ en las INSTITUCIONES ENCUESTADAS

Fuente: elaboración propia

Tanto las instituciones públicas como las privadas de la muestra cuentan con procesos o procedimientos documentados e imple102 mentados para realizar la adquisición del equipamiento biomédico. En la mayoría se emplea el análisis de costo-beneficio, donde se evalúan las bondades de la tecnología que se va a adquirir y se compara con el costo total de la inversión. La principal forma de adquisición es la asignación presupuestal, excepto en la institución A, donde emplean la figura de comodato, porque el proveedor es quien asume la responsabilidad de renovar permanentemente la tecnología.

La institución E plantea otra forma de realizar la adquisición de la tecnología biomédica; esta se propuso específicamente para los equipos de hemodiálisis, donde se cuenta con un especialista que los opera permanentemente durante todos los procedimientos, y además incluye: los consumibles para la puesta en marcha y los mantenimientos preventivos y correctivos por parte del proveedor. Esto se implementó con el fin de que un solo especialista (entrenado) opere los equipos, evitándose de esta manera que se presenten daños durante su utilización.

Algunas IPS coinciden en que la adquisición de las bombas de infusión, flujómetros y compresores vasculares, entre otros, se realice mediante el comodato, ya que después de efectuar el análisis de costo-beneficio encuentran que esta alternativa no solo permite obtener numerosas cantidades de equipos biomédicos para la atención hospitalaria, únicamente con el sostenimiento de la compra del consumible asociado para la puesta en funcionamiento de la tecnología, sino que además facilita la reposición y renovación del equipamiento para la organización a corto plazo.

En cuanto a la variable $V_{2}$ (planificación de la tecnología) se evidenció, durante la referenciación comparativa, que las seis IPS del estudio planifican la adquisición de la tecnología biomédica de acuerdo con el plan estratégico institucional, los proyectos de crecimiento, inversión, renovación y/o 
Tabla 6. Matriz de Correlación de los hospitales y las Clínicas del departamento de Antioquia

\begin{tabular}{|c|c|c|c|c|c|c|c|c|c|}
\hline Institución & 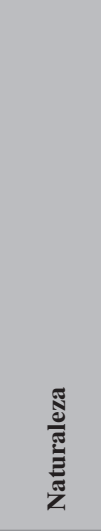 & 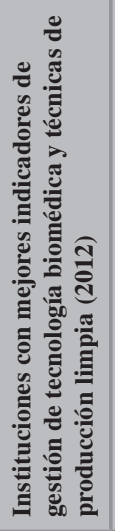 & 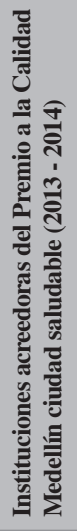 & 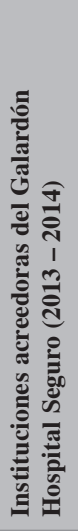 & 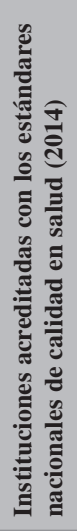 & 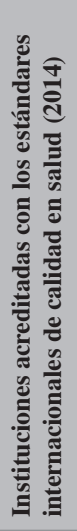 & 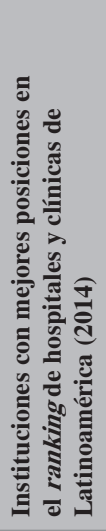 & 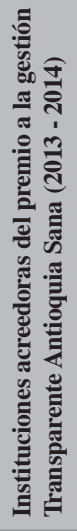 & 兴 \\
\hline Clínica Cardiovid & Privada & 0 & 0 & 0 & 0 & 0 & 1 & 0 & 1 \\
\hline $\begin{array}{l}\text { Clínica El Rosario Sede } \\
\text { Centro }\end{array}$ & Privada & 1 & 0 & 0 & 0 & 0 & 0 & 0 & 0 \\
\hline $\begin{array}{l}\text { Clínica El Rosario Sede } \\
\text { Tesoro }\end{array}$ & Privada & 1 & 0 & 0 & 0 & 0 & 0 & 0 & 0 \\
\hline Clínica Las Américas & Privada & 0 & 0 & 0 & 0 & 0 & 1 & 0 & 1 \\
\hline Clínica León XIII & Pública & 0 & 0 & 0 & 0 & 0 & 1 & 0 & 1 \\
\hline $\begin{array}{l}\text { Clínica Medellín S.A. } \\
\text { (A) }\end{array}$ & Privada & 1 & 0 & 0 & 0 & 0 & 1 & 0 & 2 \\
\hline $\begin{array}{l}\text { Clínica Oftalmológica de } \\
\text { Antioquia S.A. Clofán }\end{array}$ & Privada & 0 & 1 & 0 & 0 & 0 & 0 & 0 & 1 \\
\hline $\begin{array}{l}\text { Clínica Universitaria } \\
\text { Bolivariana }\end{array}$ & Privada & 1 & 0 & 0 & 0 & 0 & 1 & 0 & 2 \\
\hline $\begin{array}{l}\text { ESE Hospital del Sur de } \\
\text { Itagüí (B) }\end{array}$ & Pública & 0 & 0 & 0 & 1 & 0 & 0 & 1 & 2 \\
\hline $\begin{array}{l}\text { ESE Hospital General de } \\
\text { Medellín (C) }\end{array}$ & Pública & 1 & 0 & 0 & 1 & 0 & 1 & 1 & 4 \\
\hline $\begin{array}{l}\text { ESE Hospital Manuel } \\
\text { Uribe Ángel (D) }\end{array}$ & Pública & 1 & 0 & 0 & 0 & 0 & 0 & 1 & 2 \\
\hline $\begin{array}{l}\text { Fundación Colombiana de } \\
\text { Cancerología-Clínica Vida }\end{array}$ & Privada & 0 & 1 & 0 & 0 & 0 & 0 & 0 & 1 \\
\hline $\begin{array}{l}\text { Hospital Pablo Tobón } \\
\text { Uribe (E) }\end{array}$ & Privada & 1 & 0 & 0 & 1 & 0 & 1 & 0 & 3 \\
\hline $\begin{array}{l}\text { Hospital Universitario San } \\
\text { Vicente Fundación (F) }\end{array}$ & Privada & 1 & 0 & 0 & 1 & 0 & 1 & 0 & 3 \\
\hline $\begin{array}{l}\text { Unidad Hospitalaria de } \\
\text { Manrique Hermenegildo } \\
\text { de Fex }\end{array}$ & Pública & 0 & 1 & 0 & 0 & 0 & 0 & 0 & 1 \\
\hline $\begin{array}{l}\text { Unidad Hospitalaria Doce } \\
\text { de Octubre Luis Carlos } \\
\text { Galán Sarmiento }\end{array}$ & Pública & 0 & 1 & 0 & 0 & 0 & 0 & 0 & 1 \\
\hline $\begin{array}{l}\text { Unidad Prestadora de } \\
\text { Servicios de Salud San } \\
\text { Antonio de Prado }\end{array}$ & Pública & 0 & 1 & 0 & 0 & 0 & 0 & 0 & 1 \\
\hline
\end{tabular}

Fuente: elaboración propia 
reposición tecnológica. Otras necesidades son identificadas por los resultados de la evaluación que se realiza al equipamiento médico, mediante la cual se determina su obsolescencia (instituciones C y F).

Las seis IPS del estudio cuentan con un presupuesto anual que es destinado exclusivamente a la compra de equipamiento biomédico. Además, en cuanto a la elaboración del plan de adquisición, las organizaciones coinciden en que en ella participan diversas áreas, siendo la de ingeniería y mantenimiento la que más responsabilidad tiene en el proceso. En la figura 5 se describen las áreas con mayor injerencia en este proceso, según las respuestas proporcionadas por los entrevistados.

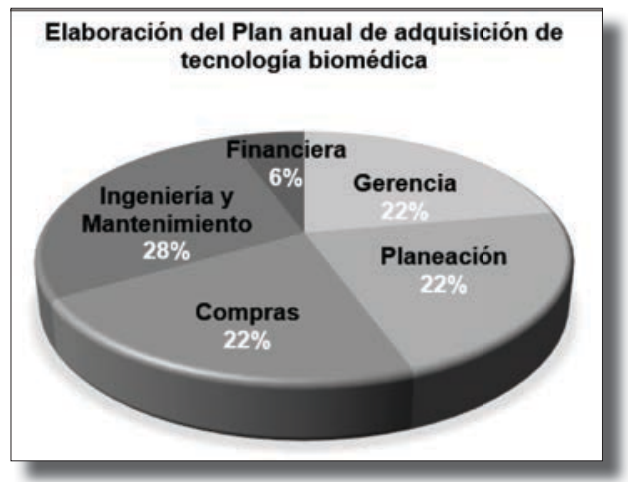

Figura 5. Porcentaje de participación de las ÁREAS EN LA ELABORACIÓN DEL PLAN DE ADQUISICIÓN

Fuente: elaboración propia

En la figura 5 se observa que las áreas de gerencia, planeación y compras de las IPS obtuvieron un $22 \%$ de participación en la relación porcentual, ya que son las encargadas de dirigir la adquisición de la tecnología según las necesidades identificadas, la planificación estratégica y la disponibilidad de recursos. El área de ingeniería y mantenimiento, con un $28 \%$, es la responsable de efectuar la evaluación técnica del equipamiento biomédico - componentes, funcionalidad, seguridad y principales riesgos, entre otros-, y el área financiera, con un $6 \%$, es la que define el estudio de factibilidad del plan y garantiza la asignación presupuestal para la compra.

Para la variable $V_{3}$ (priorización de necesidades de adquisición), los resultados obtenidos en las IPS detallan que si bien todas cuentan con procesos y procedimientos documentados e implementados para efectuar la adquisición tecnológica, en algunos casos se incorporan factores contributivos importantes para realizar la priorización de las necesidades identificadas, en tanto que en otras es difícil establecer los niveles de prioridad, porque no tienen criterios o escalas definidas.

En algunas IPS el empleo de software para la formulación de solicitudes de equipos biomédicos se ha reconocido como una buena práctica clínica, porque no solo permite consultar un archivo histórico de la organización, sino que además facilita hacer la trazabilidad de los requerimientos efectuados y medir el tiempo de respuesta en que fueron atendidos. De esta manera, se ha constituido poco a poco en una experiencia exitosa instaurada en otras instituciones del sector, al igual que la conformación de diversos comités que apoyan la gestión del ciclo de vida del equipamiento médico.

Mientras que algunos de los softwares presentados durante las entrevistas han sido comprados a los proveedores de suministros médicos, otros son el resultado de las aplicaciones desarrolladas por los ingenieros de sistemas de las IPS, constituyendo de esta manera herramientas más compactas de acuerdo con los requerimientos de cada organización y la experticia consensuada durante el proceso.

En la figura 6 se muestra que la mayor parte de las necesidades de adquisición de tecnología biomédica, según las respuestas 


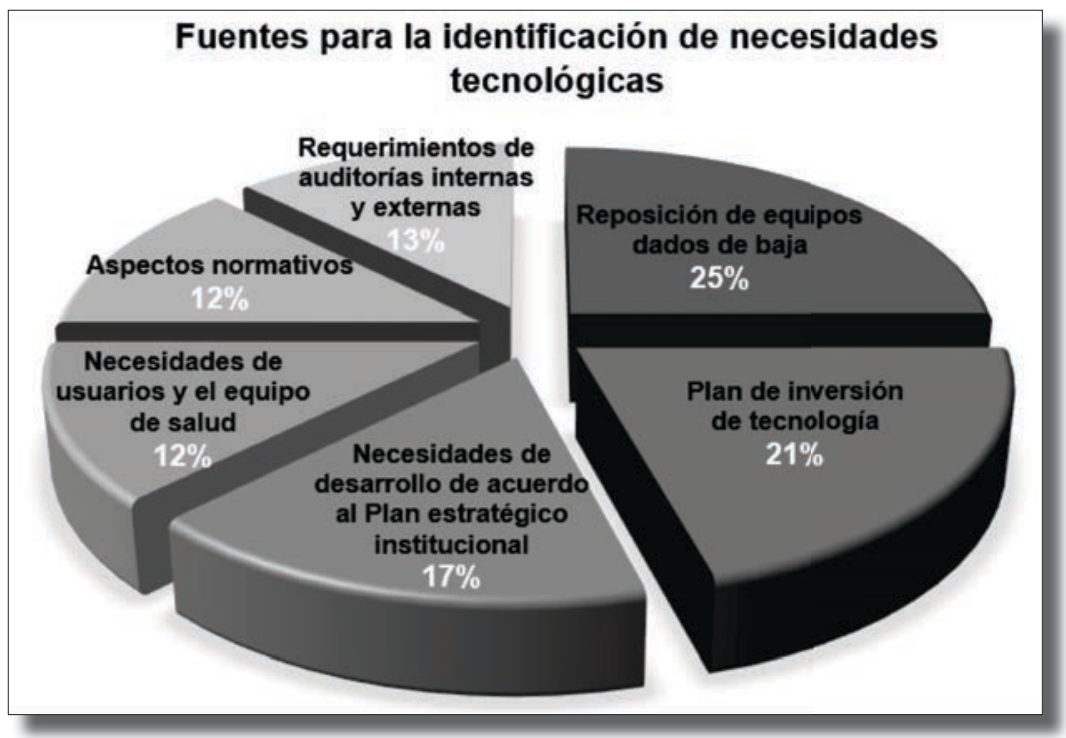

Figura 6. Principales fuentes utilizadas en las IPS

Fuente: elaboración propia

proporcionadas por los entrevistados, provienen de diversas fuentes.

En la figura 6 se observa que la principal fuente para la adquisición tecnológica es la reposición del equipo biomédico que es dado de baja. La relación porcentual obtenida para esta fuente es del $25 \%$, seguida por el plan de inversión de tecnología con un $21 \%$. Posteriormente, se sitúan las necesidades de desarrollo de acuerdo con el plan estratégico institucional, con un $17 \%$, los requerimientos de las auditorías internas y externas con un $13 \%$, y tanto los aspectos normativos como las necesidades de usuarios y el equipo de salud con el $12 \%$.

Los resultados de la variable $V_{4}$ (solicitud para la adquisición) evidencian que las seis IPS cuentan con un formato de lista de chequeo de requisitos legales y técnicos que deben cumplir los equipos biomédicos que van a ser adquiridos. La tabla 7 especifica los documentos que solicita cada institución en el momento de la adquisición de la tecnología biomédica.
En la tabla 7 se observa que la institución que cuenta con el mayor número de requisitos legales y técnicos solicita veinte documentos para la adquisición de la tecnología biomédica. Además de los documentos descritos anteriormente, la institución E exige a todos sus proveedores que hagan un curso on-line que se encuentra en la página web de la organización, con el propósito de dar a conocer sus políticas y lineamientos institucionales, y promueve que tanto las pruebas de funcionamiento como las de seguridad eléctrica y el mantenimiento preventivo del equipo biomédico se realicen en sus instalaciones, bajo la supervisión de un funcionario del área de ingeniería clínica.

La institución $\mathrm{F}$ no requiere pruebas de seguridad eléctrica, ya que estas son realizadas por su personal en el momento de adquirir la tecnología. La institución $\mathrm{C}$ cuenta con un formato denominado acta de recepción de equipos biomédicos, donde solicita el registro Invima, no solo del personal que realiza la capacitación de manejo del equipo, sino también de quien realiza el mantenimiento preventivo. 
TABla 7. Resultados Para la Variable $V_{4}$ EN LAS instituCiOnes ENCUESTADAS

\begin{tabular}{|c|c|}
\hline nología biomédica & Otros requisitos exigidos identificados en las entrevistas \\
\hline $\begin{array}{l}\text { 1. Pruebas de funcionamiento } \\
\text { 2. Pruebas de seguridad eléctrica } \\
\text { 3. Registro de mantenimiento preventivo } \\
\text { 4. Certificado de calibración (según } \\
\text { aplique) } \\
\text { 5. Registro Invima } \\
\text { 6. Registro de importación } \\
\text { 7. Manual de usuario y/o técnico } \\
\text { 8. Ficha técnica } \\
\text { 9. Certificado de garantía } \\
\text { 10. Cronograma de mantenimiento } \\
\text { preventivo } \\
\text { 11. Guía rápida de uso de la tecnología }\end{array}$ & $\begin{array}{l}\text { 1. Listado del personal capacitado en la ciudad para } \\
\text { realizar el mantenimiento preventivo y correctivo del } \\
\text { equipo } \\
\text { 2. Datos de las empresas encargadas del suministro de } \\
\text { repuestos y consumibles } \\
\text { 3. Certificado del distribuidor o representante de la casa } \\
\text { matriz de la tecnología } \\
\text { 4. Comunicado dirigido al departamento de gestión } \\
\text { humana con la disponibilidad de capacitación para el } \\
\text { personal asistencial y técnico } \\
\text { 5. Hoja de vida y registro Invima del personal técnico } \\
\text { que presta los servicios de mantenimiento y calibración } \\
\text { de los equipos con clasificaciones tipo IIb y III } \\
\text { 6. Video de capacitación de manejo del equipo } \\
\text { 7. Certificado de garantía de los repuestos durante un } \\
\text { periodo no inferior a cinco años } \\
\text { 8. Acta de entrega del equipo } \\
\text { 9. Protocolo de mantenimiento preventivo } \\
\text { 10. Hoja de vida del equipo } \\
\text { 11. Protocolo de limpieza y desinfección de la tecnología } \\
\text { 12. Cronograma de capacitación de usuario y técnico } \\
\text { 13. Registro de evaluación de la capacitación en el } \\
\text { manejo de la tecnología } \\
\text { 14. Registro de asistencia de las personas que fueron } \\
\text { capacitadas } \\
\text { 15. Requisitos de conectividad, planos de instalación y } \\
\text { necesidades de preinstalación } \\
\text { 16. Hoja de vida y registro del pago de los aportes de } \\
\text { seguridad social de las personas encargadas de realizar } \\
\text { el entrenamiento en el manejo del equipo } \\
\text { 17. Cronograma de calibración (según aplique) } \\
\text { realizar la calibración (según aplique) } \\
\text { 18. empleados para } \\
\text { adialización del equipo }\end{array}$ \\
\hline
\end{tabular}

Fuente: elaboración propia 


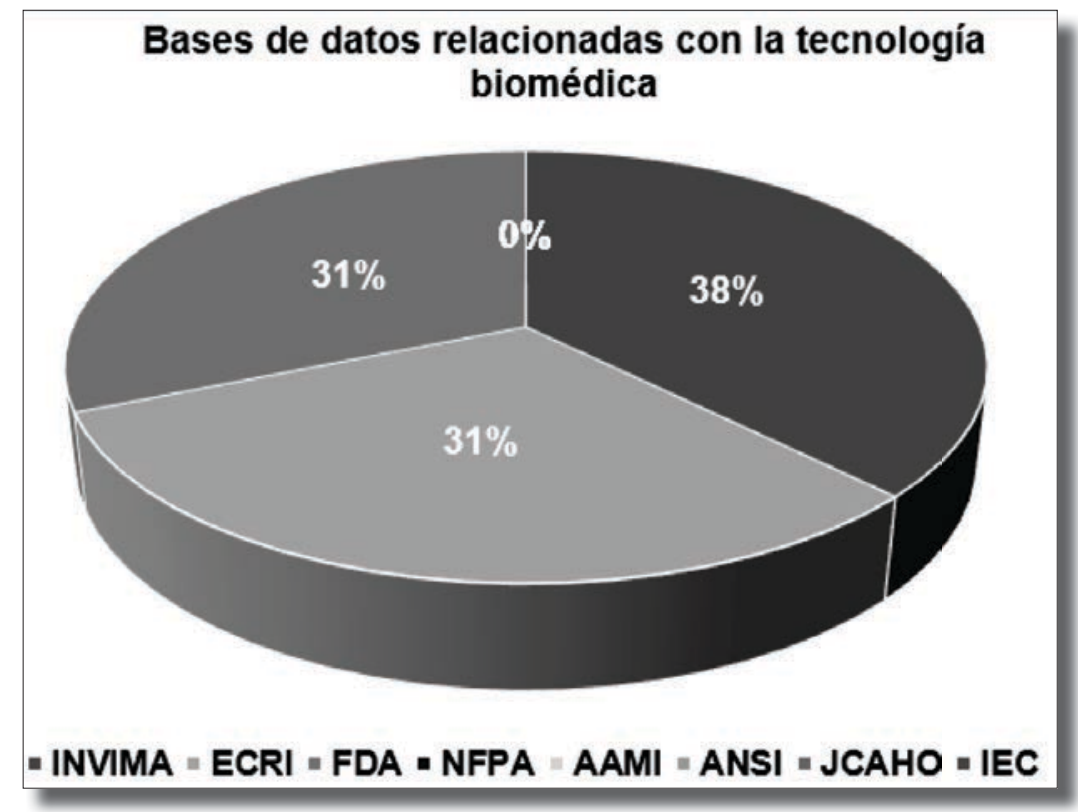

Figura 7. Proporción de búsQueda de alertas naCionales e internacionales en baSes de datos Fuente: elaboración propia

Con relación a los resultados obtenidos para la variable $V_{5}$ (análisis de las propuestas), durante las entrevistas se encontró que las seis IPS, al realizar la evaluación de la tecnología biomédica que se va adquirir, hacen búsquedas en bases de datos de las alertas nacionales e internacionales emitidas por los entes reguladores.

La figura 7 evidencia que la base de datos más empleada por las instituciones es la del Invima, con una proporción equivalente al 38\%, seguida de la del Instituto para la Investigación y Cuidados de Emergencia (ECRI) y la de la Agencia de Administración de alimentos y Drogas (FDA) con un 31\%. Las otras bases de datos no son empleadas por ninguna de las instituciones.

Para la variable $V_{6}$ (restricciones) se analizaron los aspectos que deben ser tenidos en cuenta en el momento de seleccionar una tecnología biomédica y de qué manera se garantiza que el equipamiento adquirido es compatible con los sistemas de información, conectividad, redes e infraestructura hospitalaria. En la figura 8 se observa la proporción de las restricciones que tienen mayor relevancia en las IPS para la adquisición.

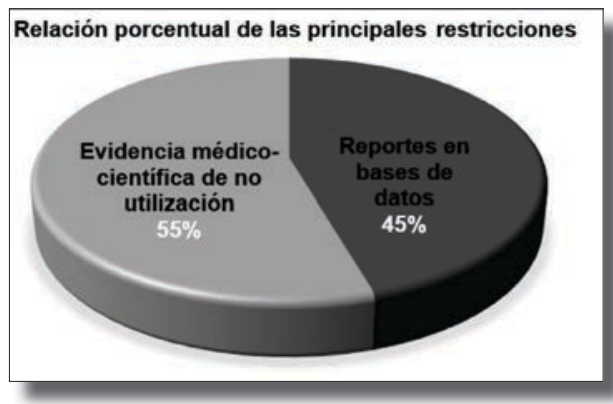

Figura 8. Principales restricciones en la ADQUISICIÓN DE TECNOLOGÍA BIOMÉDICA

Fuente: elaboración propia

En la figura 8 se muestra que la proporción de la evidencia médico-científica de no utilización para las IPS encuestadas es del 55\%, lo que la sitúa como la restricción de mayor relevancia en los procesos de adquisición del equipamiento médico. Seguidamente, 


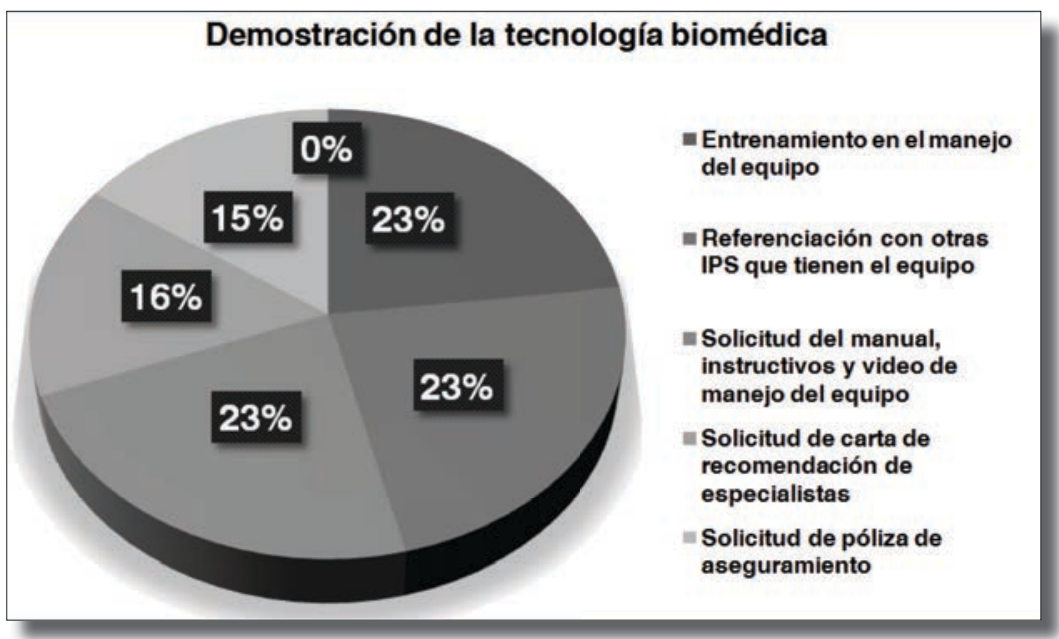

Figura 9. Porcentaje de las actividades que Se desarrollan en la demostración de la tecnología BIOMÉDICA EN LAS IPS

Fuente: elaboración propia

se encuentra la restricción de los reportes en bases de datos, con una proporción del $45 \%$.

Al indagar con los entrevistados acerca de otras posibles restricciones en el interior de las organizaciones definidas en el proceso, se encontró lo siguiente: la institución Cestableció la restricción de proveedores, debido al incumplimiento en el suministro de consumibles, repuestos o fallas detectadas durante el proceso de mantenimiento preventivo, mientras que la institución E tiene la restricción del análisis de viabilidad de la tecnología, donde se evalúan los costos de mantenimiento del equipo y las tarifas de la IPS en cada procedimiento que se va a realizar con este. Si la inversión no es costo-efectiva se toma la decisión de no adquirirlo. En la institución $\mathrm{F}$ se cuenta con la restricción presupuestal, es decir, en caso de no contar con los recursos financieros necesarios, no se compra el equipo.

Para todas las IPS, es de gran relevancia evaluar la convergencia entre los sistemas existentes, las redes y las condiciones locativas actuales con la tecnología biomédica que se encuentra en proceso de adquisición. Sin embargo, hace falta trabajar más en la definición de los criterios para evaluar la compatibilidad de las nuevas tecnologías y aquellas que se clasifican como emergentes, con los procesos, materiales, aplicaciones y dispositivos de los que disponen las organizaciones.

En la variable $V_{7}$ (demostración de la tecnología), las instituciones obtuvieron los resultados que se precisan en la figura 9, que expresa la relación porcentual para cada una de las actividades que son desarrolladas durante el proceso de demostración del equipo biomédico.

En la institución A se incluye el equipo biomédico en una póliza todo riesgo por medio de la aseguradora, desde el momento en el que el equipo ingresa a la IPS para llevar a cabo la demostración. En la institución B se solicita el diligenciamiento del formato de evaluación de tecnología por parte del personal asistencial —médicos, enfermeras y auxiliares de enfermería, entre otros.

Para iniciar el proceso de demostración en la institución E, se debe presentar una carta por parte del proveedor, en la cual se señale el tiempo de duración de esta. En la figura 9 
se observa que las actividades primordiales durante la demostración de la tecnología son el entrenamiento en el manejo del equipo, la referenciación con otras IPS que lo han adquirido con anterioridad y la solicitud del manual, instructivos y video de operación, con una relación porcentual del $23 \%$, seguidas de la solicitud de carta de recomendación de especialistas con un $16 \%$, y por último se encuentra la solicitud de pólizas de aseguramiento con un $15 \%$.

Ninguna de las IPS encuestadas realiza visitas a las instalaciones del fabricante y/o representante de la tecnología en el periodo de demostración. Aunque esta práctica no se lleva a cabo actualmente, se recomienda su incorporación, ya que permite identificar y conocer otros aspectos importantes, como por ejemplo las condiciones de almacenamiento y acondicionamiento de los equipos que posteriormente serán adquiridos por la IPS.

La variable $V_{8}$ (calidad y servicios ofrecidos) está relacionada con la disponibilidad de accesorios, consumibles, repuestos y otros servicios ofertados por el proveedor durante el tiempo de vida útil estimado para la tecnología que se pretende adquirir. Aunque se encontró que las IPS verifican la disponibilidad de repuestos y accesorios durante el tiempo de vida útil de los equipos biomédicos, por medio de los formatos que han implementado, es importante exigir al proveedor un certificado de garantía donde especifique el tiempo de representación y distribución de estos dispositivos, para asegurar su permanencia en el mercado y su fácil consecución al ser requeridos.

Para la variable $V_{9}$ (evaluación y selección final de la tecnología) se comprobó que las IPS cuentan con una metodología para realizar la evaluación de la tecnología. Aunque todas difieren en las actividades que desarrollan durante el proceso, la mayoría coincide en que participan diversas áreas y emplean formatos predefinidos para registrar el concepto técnico emitido por los responsables durante cada etapa. Se cuenta con la conformación de diversos comités en interior de las IPS que apoyan la gestión del equipo biomédico desde la adquisición hasta la disposición final, cuando han cumplido su vida útil. Además, se han implementado diversos indicadores de gestión que se miden con una periodicidad mensual y permiten conocer el desempeño del proceso y mejorarlo continuamente.

En la figura 10 se visualizan los criterios empleados en las IPS cuando se evalúa la tecnología.

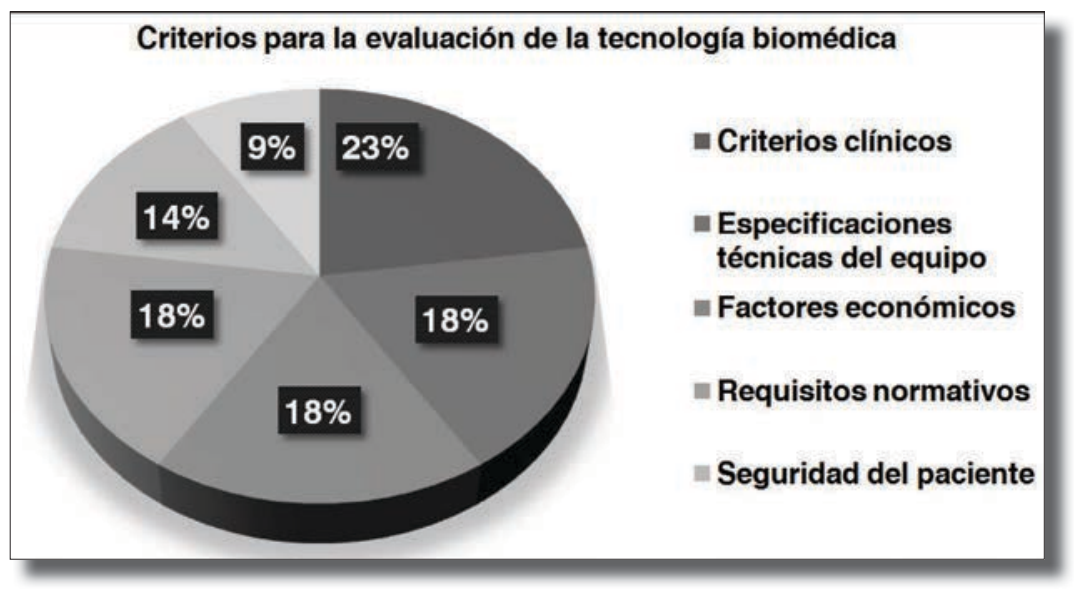

Figura 10. Criterios que emplean las IPS en la EVAluación tecnológica 
En la figura 10 se aprecia que los criterios clínicos predominan en la mayor parte de las IPS, con un porcentaje del $23 \%$, seguidos de las especificaciones técnicas, los factores económicos y los requisitos normativos con el $18 \%$; posteriormente se encuentran la seguridad del paciente con el $14 \%$ y las condiciones del mercado con el $9 \%$.

Mejores prácticas identificadas en la referenciación comparativa del proceso de adquisición de tecnología biomédica en las IPS

A continuación se presentan las mejores prácticas identificadas en el proceso de adquisición tecnológica, enfatizando en las actividades y metodologías empleadas en la evaluación de los equipos biomédicos.

La tabla 8 contiene el puntaje asignado a cada pregunta del instrumento de diagnóstico que es cuantificable, así como su corre- lación con las variables del estudio, mientras que en la tabla 9 se presentan los resultados obtenidos para cada IPS participante, ya que las preguntas cualitativas fueron analizadas en los numerales anteriores.

Además, en la tabla 9 se visualiza la sumatoria total de los puntajes resultantes para cada variable del estudio, la cual se constituye en un marco general de referencia que permite la identificación de las IPS del departamento de Antioquia con posibles mejores prácticas, según las mediciones efectuadas.

Para identificar las posibles mejores prácticas entre las IPS públicas y privadas seleccionadas se emplearon gráficos de radar. En la figura 11 se presentan los resultados obtenidos para la institución A.

La conceptualización de mejores prácticas hace referencia a un conjunto de acciones

Tabla 8. Puntaje máximo que se puede obtener en Cada Pregunta de la encuesta

\begin{tabular}{|l|c|c|c|}
\hline Número de la pregunta & Variable & Puntaje asignado & Puntaje mínimo aceptable \\
\hline $1(\mathrm{a}, \mathrm{b}, \mathrm{c}, \mathrm{d})$ & $\mathrm{V}_{1}$ & 5.0 & 3.0 \\
\hline $4(\mathrm{a}, \mathrm{b}, \mathrm{c}, \mathrm{d}, \mathrm{e})$ & $V_{2}$ & 5.0 & 3.0 \\
\hline $5(\mathrm{a}, \mathrm{b}, \mathrm{c})$ & $V_{2}$ & 5.0 & 3.0 \\
\hline $6(\mathrm{a}, \mathrm{b}, \mathrm{c})$ & $V_{3}$ & 5.0 & 3.0 \\
\hline $7(\mathrm{a}, \mathrm{b}, \mathrm{c})$ & $V_{3}$ & 5.0 & 3.0 \\
\hline $8(\mathrm{a}, \mathrm{b}, \mathrm{c}, \mathrm{d}, \mathrm{e}, \mathrm{f})$ & $V_{3}$ & 5.0 & 3.0 \\
\hline $10(\mathrm{a}, \mathrm{b}, \mathrm{c})$ & $V_{3}$ & 5.0 & 3.0 \\
\hline $11(\mathrm{a}, \mathrm{b}, \mathrm{c})$ & $V_{4}$ & 5.0 & 3.0 \\
\hline $11(\mathrm{~d}, \mathrm{e}, \mathrm{f}, \mathrm{g}, \mathrm{h}, \mathrm{i}, \mathrm{j}, \mathrm{k}, \mathrm{l}, \mathrm{m}, \mathrm{n})$ & $V_{4}$ & 5.0 & 3.0 \\
\hline $12(\mathrm{a}, \mathrm{b}, \mathrm{c})$ & $V_{5}$ & 5.0 & 3.0 \\
\hline $12(\mathrm{~d}, \mathrm{e}, \mathrm{f}, \mathrm{g}, \mathrm{h}, \mathrm{i}, \mathrm{j}, \mathrm{k})$ & $V_{5}$ & 5.0 & 3.0 \\
\hline $12(\mathrm{~m}, \mathrm{n}, \mathrm{o})$ & $V_{5}$ & 5.0 & 3.0 \\
\hline $13(\mathrm{a}, \mathrm{b}, \mathrm{c}, \mathrm{d}, \mathrm{e}, \mathrm{f})$ & $V_{6}$ & 5.0 & 3.0 \\
\hline $15(\mathrm{a}, \mathrm{b}, \mathrm{c}, \mathrm{d}, \mathrm{e}, \mathrm{f})$ & $V_{7}$ & 5.0 & 3.0 \\
\hline $16(\mathrm{a}, \mathrm{b}, \mathrm{c})$ & $V_{8}$ & 5.0 & 3.0 \\
\hline $20(\mathrm{a}, \mathrm{b}, \mathrm{c}, \mathrm{d}, \mathrm{e}, \mathrm{f})$ & $V_{9}$ & 5.0 & 3.0 \\
\hline
\end{tabular}


Tabla 9. Mejores Prácticas identificadas en las IPS encuestadas

\begin{tabular}{|c|c|c|c|c|c|c|c|c|}
\hline \multirow{2}{*}{ 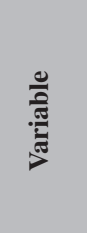 } & \multirow{2}{*}{ 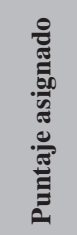 } & \multirow{2}{*}{ 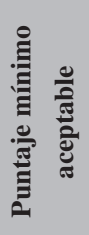 } & \multicolumn{6}{|c|}{ Institución } \\
\hline & & & $\mathbf{A}$ & B & C & D & $\mathbf{E}$ & $\mathbf{F}$ \\
\hline$V_{1}$ & 5.0 & 3.0 & 2.5 & 1.2 & 2.5 & 2.5 & 2.5 & 2.5 \\
\hline$V_{2}$ & 5.0 & 3.0 & 3.0 & 4.0 & 5.0 & 4.0 & 4.0 & 4.0 \\
\hline$V_{3}$ & 5.0 & 3.0 & 5.0 & 5.0 & 4.8 & 4.3 & 4.2 & 4.2 \\
\hline$V_{4}$ & 5.0 & 3.0 & 4.3 & 4.7 & 4.7 & 4.7 & 4.7 & 4.5 \\
\hline$V_{5}$ & 5.0 & 3.0 & 3.7 & 4.0 & 4.0 & 3.7 & 4.0 & 4.0 \\
\hline$V_{6}$ & 5.0 & 3.0 & 1.7 & 2.5 & 1.7 & 1.7 & 1.7 & 0.8 \\
\hline$V_{7}$ & 5.0 & 3.0 & 3.3 & 4.2 & 4.2 & 3.3 & 3.3 & 3.3 \\
\hline$V_{8}$ & 5.0 & 3.0 & 5.0 & 5.0 & 5.0 & 5.0 & 5.0 & 5.0 \\
\hline$V_{9}$ & 5.0 & 3.0 & 1.7 & 5.0 & 3.3 & 0.8 & 4.2 & 2.5 \\
\hline$\sum$ total & 45.0 & 27.0 & 30.2 & 35.6 & 35.2 & 30.0 & 33.6 & 30.8 \\
\hline
\end{tabular}

Fuente: elaboración propia

que han proporcionado un buen o incluso excelente resultado en un contexto específico, y se espera que en condiciones equivalentes se puedan alcanzar logros similares (24).

Se considera que una institución alcanza la mejor práctica para cada variable del estudio cuando obtiene el mayor puntaje sobre su grupo. Esto quiere decir que el valor resultante no tiene que ser 5.0 necesariamente, ya que este corresponde a una práctica ideal.

Una práctica ideal se caracteriza por llevar a cabo un procedimiento idóneo, constituyéndose en la metodología más apropiada y recomendable para ser adoptada en otros contextos.

En el gráfico de radar presentado en la figura 11 se observa que los factores críticos de éxito para la institución A se reflejan en las variables $V_{1}, V_{3}$ y $V_{8}$ las cuales obtienen la calificación de 2.5 puntos para la primera y 5.0 puntos para las demás, lo que representa que ha alcanzado las posibles mejores prácticas de las variables en mención. Estos datos permiten establecer un parámetro global de referencia para la variable $V_{3}$, cuyo resultado fue de 4.6 para el grupo completo de las IPS con un valor mínimo de 4.2. En relación con la variable $V_{l}$ se evidencia que la media ponderada es de 2.3 y el valor mínimo obtenido de 1.2 , mientras que para $V_{8}$ todas las organizaciones alcanzaron el máximo puntaje asignado. En la figura 12 se visualizan los resultados para la institución B, la cual obtuvo las posibles mejores prácticas, en comparación con las otras IPS, en las variables $V_{4}, V_{5}, V_{6}, V_{7}$ y $V_{9}$.

Para la institución B, en el gráfico de radar se observa que los factores críticos de éxito predominan en las variables $V_{4}, V_{5}, V_{6}, V_{7}$ y $V_{9}$, por contar con el mayor puntaje sobre el grupo completo de las IPS. Los resultados de $V_{4}$ fueron iguales a los de las instituciones C, D y E, lo que permite establecer un parámetro global de referencia para esta variable, teniendo en cuenta que la media ponderada es de 4.6 y el valor mínimo alcanzado fue de 4.3. Este caso es similar a los resultados de 


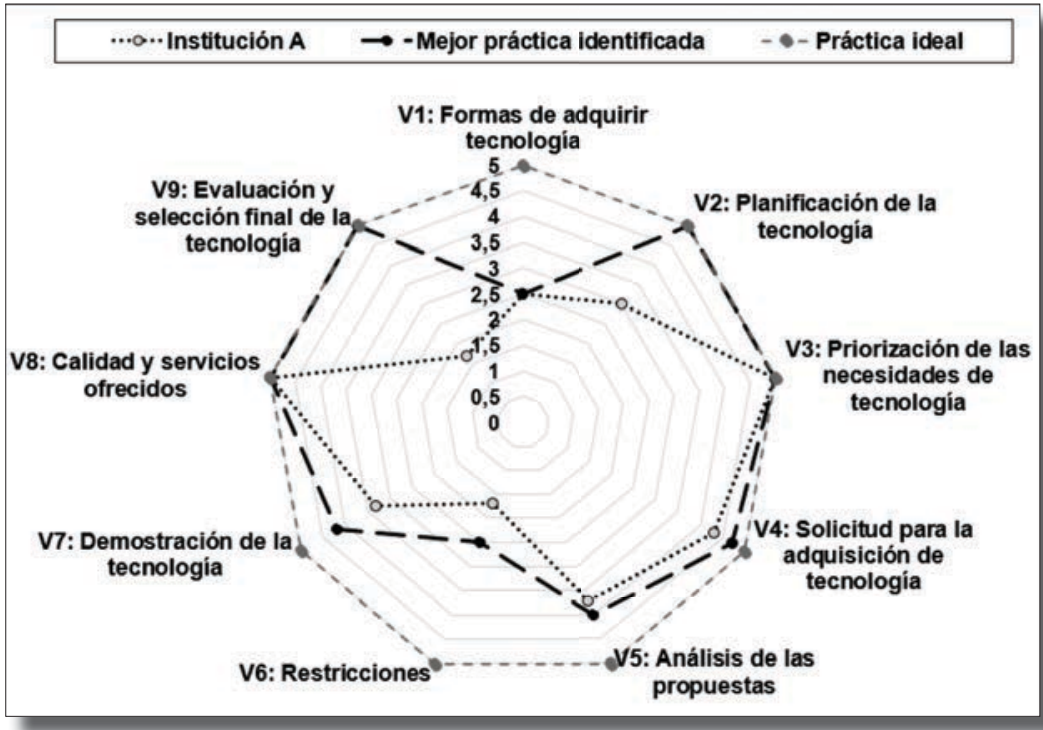

Figura 11. Gráfico de radar de los resultados para la institución A

Fuente: elaboración propia

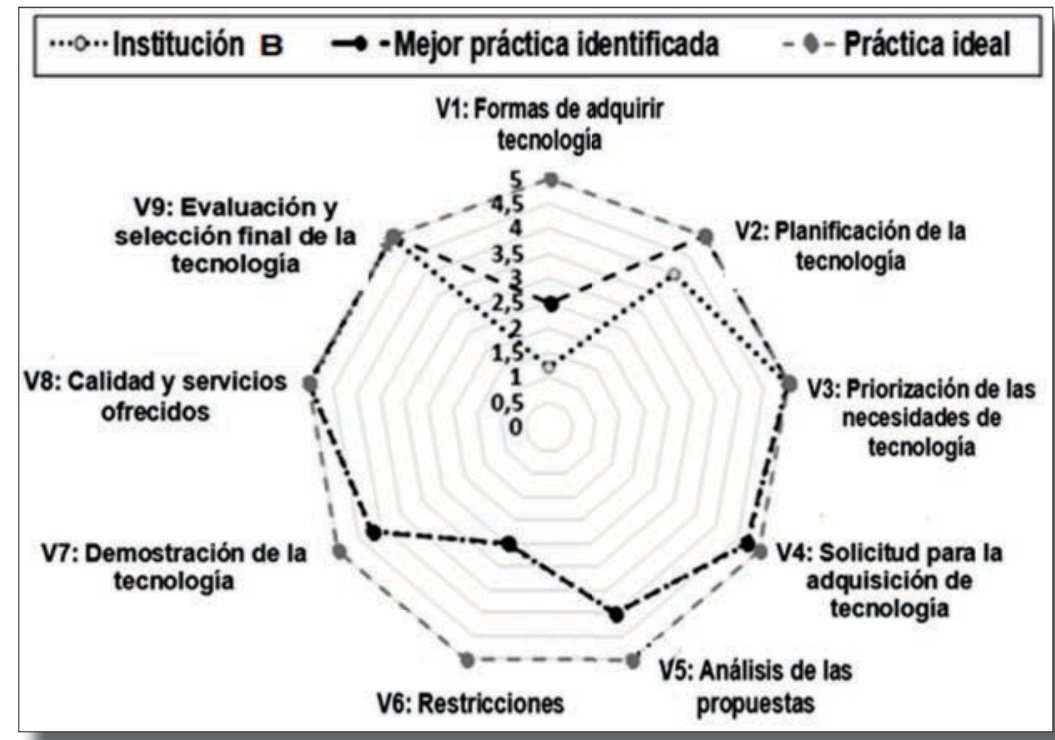

Figura 12. Gráfico de radar de los resultados para la institución B

Fuente: elaboración propia 
la variable $V_{5}$, en el cual las instituciones $\mathrm{C}$, E y F obtuvieron un puntaje de 4.0, constituyéndose como un patrón global de referencia donde la media ponderada es de 3.9 y el puntaje mínimo alcanzado es de 3.7. En $V_{6}$ la institución B alcanzó 2.5 puntos, lo cual la sitúa como poseedora de posibles mejores prácticas para esta variable, en comparación con las demás IPS. Para la variable $V_{7}$ obtuvo un puntaje de 4.2, al igual que la institución C. Para la $V_{9}$, obtuvo 5.0 puntos, lo que indica que efectúa un procedimiento idóneo y apropiado para ser adoptado en otros posibles contextos del sector. En la figura 12 se observa que la institución B es considerada poseedora de las posibles mejores prácticas de las IPS públicas, ya que la sumatoria total del puntaje obtenido en la tabla 8 es de 35.6, y este supera los valores adquiridos por las demás organizaciones.

En el gráfico de radar correspondiente a la institución $\mathrm{C}$ se evidencia que los factores críticos de éxito prevalecen en las variables $V_{1}, V_{2}, V_{4}, V_{5}$ y $V_{7}$, según los puntajes obtenidos para cada una de estas. $\mathrm{Al}$ analizar las variables por separado se encuentra que para la $V_{1}$ obtuvo el mismo puntaje que las instituciones A, D, E y F; en cuanto a $V_{2}$ fue la única en alcanzar una calificación de 5.0 puntos. Para las variables $V_{4}, V_{5}$ y $V_{7}$ consiguió igual puntaje que otras organizaciones (ver los resultados de la figura 13).

Según la figura 14, la institución D, para las variables $V_{1}$ y $V_{4}$ fue una de las IPS que alcanzaron el mayor puntaje (ver resultados de las figuras 11 y 12).

En la figura 15 se encuentran los hallazgos para la institución E, la cual es considerada poseedora de las posibles mejores prácticas de las IPS privadas, ya que la sumatoria total del puntaje obtenido es mayor que el adquirido por las demás.

De acuerdo con el gráfico de radar de la institución E que se visualiza en la figura 15, se observa que los factores críticos de éxito predominan en las variables $V_{1}, V_{4}$ y $V_{5}$ por obtener los mayores puntajes.

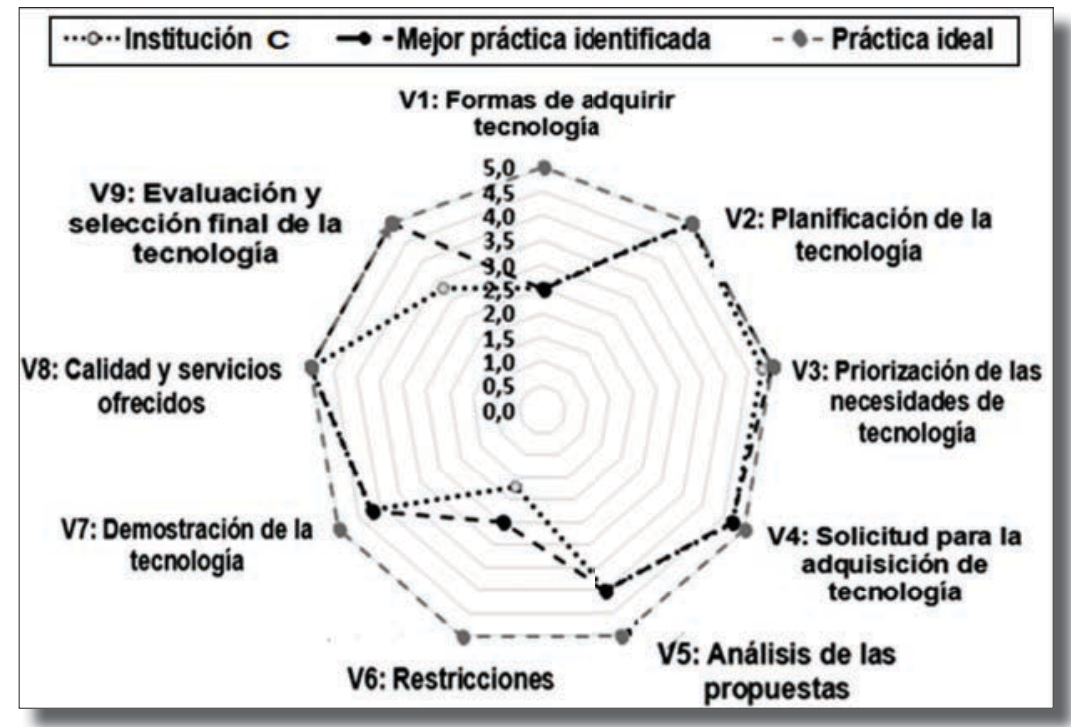

Figura 13. Gráfico de Radar de los resultados para la institución C 


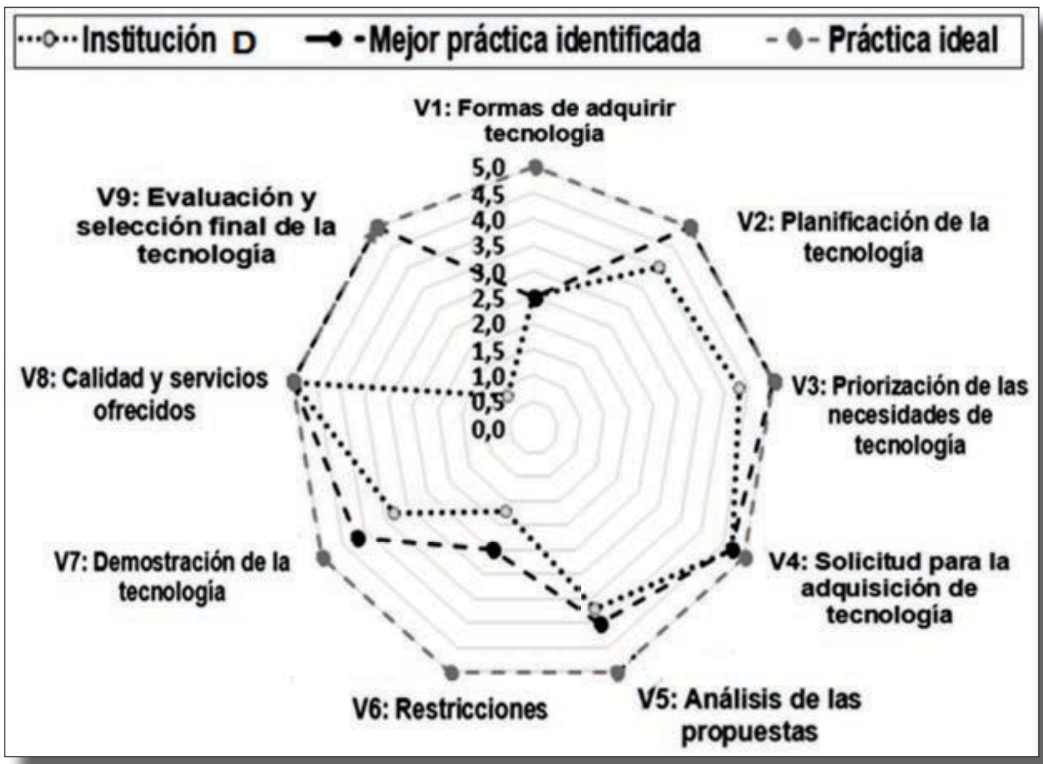

Figura 14. Gráfico de Radar de los Resultados para la institución D

Fuente: elaboración propia

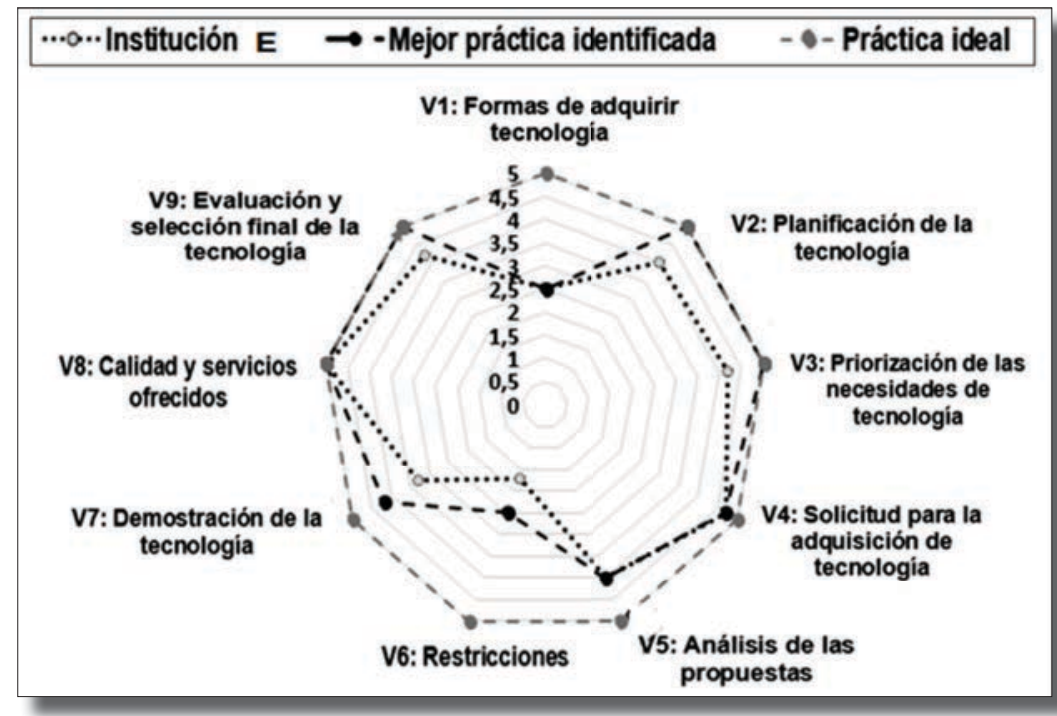

Figura 15. Gráfico de radar de los resultados para la institución E

Fuente: elaboración propia

Según la figura 16, en la institución F los factores críticos de éxito predominan en las variables $V_{1}$ y $V_{5}$ por lograr los mayores puntajes. De acuerdo con los resultados, se evi114 dencia que las IPS cada vez se preocupan más por mejorar la calidad en la prestación de sus servicios mediante la gestión por procesos. Este es considerado uno de los principales factores que han llevado a las organizaciones a buscar una posible mejor práctica en cualquier otro lugar. Sin embargo, hace falta incorporar estos ejercicios de referenciación 


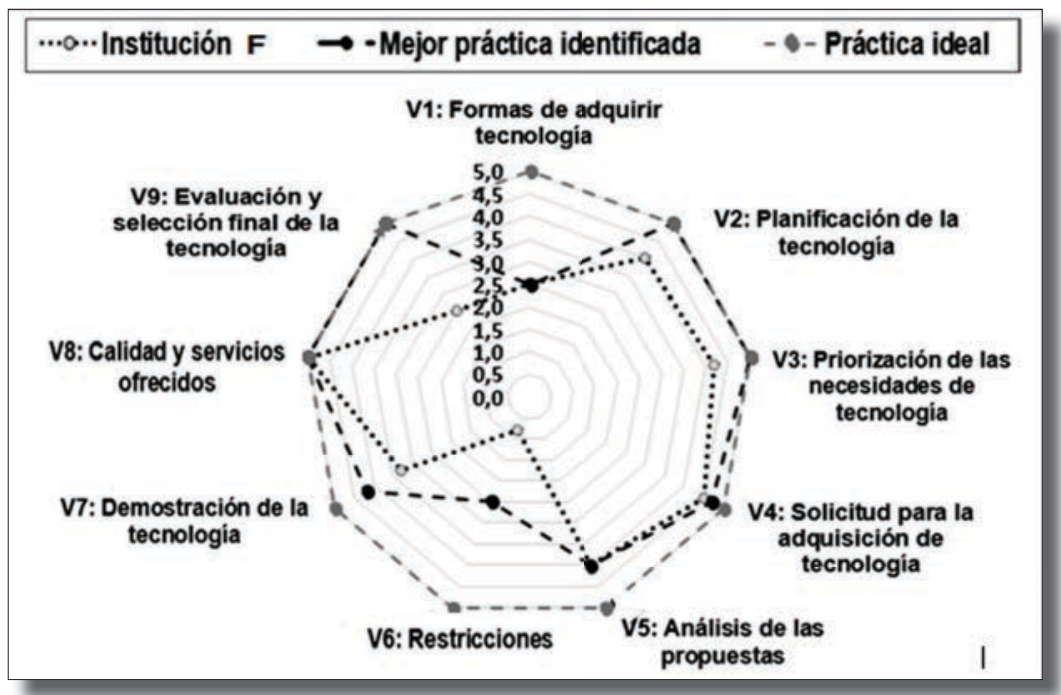

Figura 16. Gráfico de radar de los resultados para la institución F

Fuente: elaboración propia

comparativa en el sector hospitalario, como una oportunidad para que la innovación, el desarrollo y la creatividad que se manifiestan en las IPS, sean evaluados, analizados e instaurados por otras organizaciones decididas a incrementar sus niveles de competitividad y que han optado por alcanzar altos estándares para la atención integral en salud.

\section{Discusión}

De acuerdo con los anteriores resultados, se evidencia que hace falta incorporar herramientas o metodologías para ejecutar la priorización de las necesidades de adquisición tecnológica de manera sistemática, facilitando la toma de decisión con respecto a la presencia de numerosas solicitudes de compra de equipos médicos. Esto permite establecer el orden jerárquico que debe seguirse para realizar las adquisiciones, de tal manera que no se genere el cierre de servicios asistenciales por falta de equipamiento.

En la Empresa Social del Estado (ESE) Hospital La María (IPS de alta complejidad), plantean un método de evaluación de equipamiento médico compuesto por tres fases, una de las cuales consiste en la ejecución de un estudio exploratorio en el que se aplican cuestionarios a los ingenieros clínicos de las IPS, para conocer cómo ha sido su experiencia en relación con la adquisición de una tecnología médica específica (25).

Así mismo, dentro de las etapas de la metodología de evaluación de la tecnología biomédica que realiza el Hospital Infantil Consejo de Medellín (IPS de mediana-alta complejidad), se hace un ejercicio de benchmarking tecnológico, que se basa en la ejecución de tres visitas técnicas a otras IPS que disponen de la marca y el modelo del equipo que se evalúa en sus instalaciones (26). Estas actividades, no solo permiten la transferencia de conocimiento entre las instituciones de salud, sino que además promueven la adopción de sus aprendizajes organizacionales. Es conveniente que las actividades y los informes que se elaboran al evaluar los equipos biomédicos sean divulgados a otras IPS, o que se cuente con mecanismos (foros tecnológicos, mesas redondas, debates, 
entre otros) donde puedan compartirse las experiencias exitosas que se han tenido con algún tipo de tecnología, contribuyendo así al establecimiento de evidencia científica que promueva la construcción de políticas y lineamientos en el área de la ingeniería clínica en el país.

Según los resultados de una encuesta que fue aplicada en once IPS de la ciudad de Cali (seis IPS de alta complejidad, dos de mediana-alta, dos de mediana y una de baja complejidad), se comprueba que algunas organizaciones cuentan con metodologías de evaluación del equipamiento médico que son implementadas de manera previa a su adquisición, y que además incorporan la búsqueda activa en las bases de datos de las agencias nacionales e internacionales y de otras instituciones tales como el Invima, la Red Nacional de Tecnovigilancia y la FDA. Otra información relevante que es tenida en cuenta para la toma de decisiones en el proceso de adquisición tecnológica en estas organizaciones se encuentra relacionada con el servicio de mantenimiento preventivo, la consecución de los repuestos del equipamiento médico por un periodo no inferior a diez años y el conjunto de especificaciones clínicas (27). Esto demuestra que no solo a nivel del departamento de Antioquia, las IPS han comenzado a preocuparse por abordar el problema de la información imprecisa e insuficiente en el momento de evaluar las opciones tecnológicas, ya que poco a poco han incorporado metodologías que no solo tienen en cuenta el costo de adquisición del equipo, como sucedía hace algunos años atrás. Mediante la aplicación de estudios como este, se evidencia cómo las IPS han adoptado criterios de tipo técnico, clínico y financiero, con el fin de proporcionar la cobertura a todas las necesidades tecnológicas locales y minimizar el efecto de la subjetividad en la toma de decisiones durante el proceso de adquisición.

\section{Conclusiones}

En Colombia no se cuenta con un manual o guía metodológica estandarizada que contenga tanto los requisitos técnicos como los requisitos legales que deben ser tenidos en cuenta para efectuar la evaluación de la tecnología biomédica que es adquirida, lo cual no permite que las IPS dispongan de un marco general de referencia para homologar su práctica clínica. Con la realización de este tipo de estudios se promueve el establecimiento de políticas nacionales con base en las mejores prácticas identificadas, de tal manera que se fortalezca la gestión de las necesidades tecnológicas en el interior de las organizaciones y se apoye la toma de decisiones en torno a la evaluación de los dispositivos y equipos.

La gestión de la tecnología biomédica en las instituciones hospitalarias ha impulsado la implementación de procesos y procedimientos para efectuar la adquisición del equipamiento médico. En algunas, se han incorporado factores contributivos importantes para realizar la priorización de las necesidades identificadas, en tanto que en otras es difícil establecer los niveles de prioridad porque no cuentan con criterios o metodologías definidas.

Las necesidades de adquisición de tecnología biomédica en una institución de salud son el resultado de la demanda actual en la prestación de los servicios, y si bien las organizaciones han comenzado a definir metodologías para realizar una evaluación adecuada, aún existe cierto grado de subjetividad en las decisiones que se toman con relación a la selección de la mejor alternativa tecnológica dentro de un conjunto de opciones.

Con el paso de los años se han interiorizado criterios, tanto técnicos como clínicos, de seguridad y económicos, alrededor de la evaluación de la tecnología biomédica, debido a que las instituciones han tomado 
conciencia de la importancia de la implementación de metodologías de análisis que permitan la justificación y validez de la toma de decisiones. Sin embargo, el proceso cada vez se dificulta más con la incorporación de tecnologías emergentes y la multiplicidad de alternativas presentes en el mercado.

Se considera que la aplicación del ejercicio de referenciación comparativa fue adecuada para identificar y analizar las posibles mejores prácticas de las IPS, tanto públicas como privadas, del departamento de Antioquia, ya que permitió analizar los aprendizajes obtenidos por las organizaciones al efectuar la evaluación de la tecnología biomédica en el marco del proceso de adquisición.

A lo largo de la investigación, se encontró que la IPS pública que posee mejores prácticas en relación con las variables analizadas es la ESE Hospital del Sur de Itagüí, por contar con procesos muy estandarizados para efectuar la priorización de las necesidades de tecnología biomédica, de acuerdo con los requisitos técnicos, legales y los propios de la organización. Otro de los aspectos que es preciso resaltar de esta IPS es la metodología empleada para realizar la evaluación y selección final del equipamiento que se pretende adquirir, ya que entre el grupo de organizaciones del estudio fue la que alcanzó el máximo puntaje del estándar, reflejando ello su compromiso en el mejoramiento continuo de sus procesos.

En el grupo de las IPS privadas se identificó que los factores críticos de éxito en el proceso de adquisición del equipamiento están relacionados con la debida planeación de la compra de tecnología y el análisis de las propuestas que realiza el grupo de expertos. Este es el caso del Hospital Pablo Tobón Uribe y del Hospital Universitario San Vicente Fundación, que obtuvieron los mayores puntajes para estas variables del estudio en comparación con las demás.
Se evidencia que las organizaciones han alcanzado mejores niveles de desempeño en la gestión de la tecnología biomédica, ya que han desarrollado aplicaciones propias, o incluso han incorporado diversos tipos de software en la administración del equipamiento. Esto se pudo comprobar durante las entrevistas realizadas al grupo de expertos, por medio del cumplimiento de las metas establecidas para cada uno de sus indicadores de gestión.

La metodología empleada en este estudio provee un nivel de referencia de las instituciones del sector hospitalario, posibilitando su caracterización, una mayor comprensión del entorno, y a la vez se constituye en una oportunidad de transferencia de conocimiento, mediante los aprendizajes adquiridos de la experiencia de los competidores.

$\mathrm{Al}$ caracterizar la situación actual de las instituciones hospitalarias de la ciudad de Medellín se identificaron las mejores prácticas, tanto para las IPS públicas como para las privadas, y se evidenció que la gestión por procesos contribuye al logro de sus objetivos estratégicos y las ha llevado a posicionarse dentro del sector como IPS sólidas y comprometidas con la seguridad del paciente. Este ejercicio no solo permitió conocer los aprendizajes obtenidos por las organizaciones del sector, sino que además reconoció sus factores críticos de éxito, referenciando la experticia y el conocimiento adquirido de las formas organizativas de trabajo de cada una de estas, específicamente en lo concerniente a la evaluación de la tecnología biomédica durante el proceso de adquisición.

Solo en la medida en que los factores críticos de éxito sean socializados con las otras instituciones del área hospitalaria, y se cuente con la apertura suficiente para aprender de quienes han alcanzado niveles superiores de desempeño, se podrá efectuar el cierre del ciclo de reconocimiento, adaptación y adopción de mejores prácticas clínicas. 
Se concluye que este ejercicio de referenciación comparativa es una oportunidad de transferencia de conocimiento, ya que permite el intercambio de experiencias y de aprendizajes organizacionales, así como la identificación de oportunidades de mejoramiento, al tener la posibilidad de compararse con las mejores prácticas evidenciadas. Esto contribuye al desarrollo de innovaciones en los procesos y a la identificación de los factores críticos de éxito que permiten potenciar y alcanzar altos niveles de crecimiento.

\section{Referencias bibliográficas}

1. Organización Mundial de la Salud. Guía de recursos para el proceso de adquisición de dispositivos médicos. Ginebra: Organización Mundial de la Salud; 2012.

2. Cruz AM. Gestión tecnológica hospitalaria. Un enfoque sistémico. Bogotá: Editorial Universidad del Rosario; 2010.

3. Valencia J, Manrique R. Evaluación de tecnologías en salud. CES Medicina. 2004; 18(2):81-86.

4. Ayres M. Evaluación económica de tecnologías médicas. Revista de Antiguos Alumnos del IEEM. 2006; 9(13):79-87.

5. Farzipoor Saen R. Technology selection in the presence of imprecise data, weight restrictions, and nondiscretionary factors. The International Journal of Advanced Manufacturing Technology. 2009; 41(7-8):827-838.

6. Carvajal M, Ruiz CG. Evaluación técnica y clínica de tecnología biomédica en procesos de adquisición: Un enfoque en evaluación de tecnologías en salud. Revista Ingeniería Biomédica. 2008; 2(4):34-45.

7. Organización Mundial de la Salud. Formulación de políticas sobre dispositivos médicos. Ginebra: Organización Mundial de la Salud; 2012.

8. Organización Mundial de la Salud. Evaluación de las necesidades de dispositivos médicos. Ginebra: Organización Mundial de la Salud; 2012.

9. Otálvaro EH. Modelo de evaluación y gestión de equipamiento. Documento 5088. Bogotá: Ministerio de Protección Social; 2007.

10. Franco R, Vilcahuamán L. Ingeniería clínica y su relación con la epidemiología. Revista Peruana de Epidemiología. 2010; 14(1):6-16.

11. Ranking de los mejores hospitales y clínicas de América Latina [Internet]. Disponible en: http:// rankings.americaeconomia.com/mejores-clinicashospitales-2014/ranking/

12. Ríos RM. Seguimiento, medición, análisis y mejora en los sistemas de gestión. Enfoque bajo indicadores de gestión y balanced scorecard. Bogotá: Icontec; 2009.
13. Salazar KJ. Contribución metodológica para la evaluación de la tecnología biomédica en la Clínica Universitaria Bolivariana con fines de acreditación según los estándares de la Joint Commission International [tesis de maestría]. Medellín: Universidad Nacional de Colombia; 2015.

14. Malhotra NK. Investigación de mercados. Quinta edición. México: Pearson Educación; 2008.

15. Biogestión, Cigraf. Determinación de la capacidad de integración empresarial y la evaluación tecnológica y su implementación en el Centro de Desarrollo Tecnológico para la Competitividad de la Industria de la Comunicación Gráfica Cigraf. Bogotá: Universidad Nacional de Colombia; 2006.

16. Kotler P, Armstrong G. Marketing. Versión para Latinoamérica. Decimoprimera edición. México: Pearson Educación; 2007.

17. Parada L, Taborda A, Chicaíza L. Evaluación económica de tecnología sanitaria y toma de decisiones en salud. Coyuntura Económica: Investigación Económica y Social. 2013; XLIII(2):81-95.

18. Gutiérrez C, Giedion U, Muñoz A, Ávila A. Series de notas técnicas sobre procesos de priorización de salud. Procesos de priorización explícita en salud: un enfoque sistémico. Bogotá: Banco Interamericano de Desarrollo; 2012.

19. Rodríguez ED. Ingeniería clínica. La Habana, Cuba: Instituto Superior Politécnico José Antonio Echeverría. Centro de Bioingeniería Cebio; 2003.

20. González B. La incorporación de nuevas tecnologías en el Sistema Nacional de Salud. Costeefectividad y presiones sobre el gasto sanitario. Presupuesto y Gasto Público. 2007; 49:87-105.

21. Molina T. Ingeniería clínica para no ingenieros: adquisición de equipos médicos. Revista Ingeniería Biomédica. 2007; 2:40-47.

22. Rosales A, González R, Cantillo E, Esquivel L, Rodríguez M. Metodología para la evaluación de tecnologías de salud en el Seguro Social de Costa Rica, 2014.

23. Schonfeld C. La evaluación de tecnologías en salud como herramienta para la mejora de la gestión del laboratorio. Acta Bioquímica Clínica Latinoamericana. 2013; 47(1): 121-143.

24. Naranjo D, Ruiz D. Aplicación del benchmarking en la gestión de la cadena de aprovisionamiento sanitaria: efectos sobre el coste y la calidad de las compras. Gaceta Sanitaria. 2015; 29(2):118-122.

25. Hernández AM, Ruiz LF, Duque CM, Álzate JI, García JH. Modelo para evaluación de tecnología biomédica y su aplicación en la adquisición de equipos para un hospital público colombiano. En Folgueras J, coordinador. IFMBE Proceedings 33. La Habana, 2011. p. 444-7.

26. Camacho J. Modelo de gestión de tecnología biomédica para una institución de salud de tercer nivel [tesis de maestría]. Medellín: Instituto Tecnológico Metropolitano; 2015.

27. González A, Collazos M, García LJ, Ladino JA, Cano A, González SA. Análisis del estado actual de la ingeniería clínica en las instituciones hospitalarias de Cali. Revista Ingeniería Biomédica. 2015; 9(18): 73-80. 